\title{
Model-independent sensitivity estimates for the electromagnetic dipole moments of the $\tau$-lepton at the CLIC
}

\author{
M. Köksal, ${ }^{1, *}$ A. A. Billur, ${ }^{2, \dagger}$ A. Gutiérrez-Rodríguez, ${ }^{3, \neq}$ and M. A. Hernández-Ruíz, ${ }^{4,8}$ \\ ${ }^{1}$ Department of Optical Engineering, Cumhuriyet University, 58140, Sivas, Turkey \\ ${ }^{2}$ Department of Physics, Cumhuriyet University, 58140, Sivas, Turkey \\ ${ }^{3}$ Facultad de Física, Universidad Autónoma de Zacatecas, \\ Apartado Postal C-580, 98060 Zacatecas, México \\ ${ }^{4}$ Unidad Académica de Ciencias Químicas, Universidad Autónoma de Zacatecas, \\ Apartado Postal C-585, 98060 Zacatecas, México
}

(Received 5 April 2018; published 12 July 2018)

\begin{abstract}
We establish model-independent sensitivity on the anomalous magnetic and electric dipole moments of the $\tau$-lepton using the two-photon processes $\gamma \gamma \rightarrow \tau^{+} \tau^{-}$and $\gamma \gamma \rightarrow \tau^{+} \tau^{-} \gamma$. We use $\mathcal{L}=10,50,100,300$, $500,1000,1500,2000,3000 \mathrm{fb}^{-1}$ data collected with the future $e^{+} e^{-}$linear collider such as the CLIC at $\sqrt{s}=380,1500,3000 \mathrm{GeV}$, and we consider systematic uncertainties of $\delta_{\text {sys }}=0 \%, 3 \%, 5 \%$. We obtain sensitivity estimates at $95 \%$ C.L. on the anomalous dipole moments to the $\tau$-lepton $-0.00015 \leq \tilde{a}_{\tau} \leq$ 0.00017 and $\left|\tilde{d}_{\tau}(e \mathrm{~cm})\right|=9.040 \times 10^{-19}$ that may be achievable in future experiments. Our results show that the processes under consideration are very promising for probing the dipole moments of the $\tau$-lepton at the future $e^{+} e^{-}$linear collider at the $\gamma \gamma$ mode.
\end{abstract}

DOI: $10.1103 /$ PhysRevD.98.015017

\section{INTRODUCTION}

One of the greatest achievements of the Standard Model (SM) [1-3] is the extremely precise measurement of the electric (EDM) and magnetic (MM) dipole moments of the electron and muon $g-2[[4],[5]]$,

$$
\begin{array}{r}
a_{e}^{\text {Exp }}=1159652180.73(28) \times 10^{-12} \quad[0.24 \mathrm{ppb}], \\
a_{\mu}^{\text {Exp }}=11659209.1(5.4)(3.3) \times 10^{-10} \quad[0.54 \mathrm{ppm}],
\end{array}
$$

respectively. The theoretical prediction of the SM [6] is given by

$$
a_{\mu}^{\mathrm{SM}}=116591803(1)(42)(26) \times 10^{-11} .
$$

On the other hand, compared to the electron or muon mass, the $\tau$-lepton has a large mass of $m_{\tau}=1776.82 \pm$ $0.16 \mathrm{MeV}$ [6]. This allows the expectation of an essential

\footnotetext{
mkoksal@cumhuriyet.edu.tr

†abillur@cumhuriyet.edu.tr

alexgu@fisica.uaz.edu.mx

${ }^{\S}$ mahernan@uaz.edu.mx
}

Published by the American Physical Society under the terms of the Creative Commons Attribution 4.0 International license. Further distribution of this work must maintain attribution to the author(s) and the published article's title, journal citation, and DOI. Funded by SCOAP ${ }^{3}$. enhancement in the sensitivity to the effects of new physics beyond the Standard Model (BSM), such as its dipole moments [7]. However, the very short lifetime of this unstable particle makes it impossible to directly measure its electromagnetic properties. Indirect information must therefore be obtained by precisely measuring cross sections and decay rates in processes involving the emission of a real photon by the $\tau$-lepton.

With respect to the anomalous magnetic moment of the $\tau$-lepton, the $\mathrm{SM}$ prediction is $a_{\tau}^{\mathrm{SM}}=117721(5) \times 10^{-8}$ $[8,9]$ and the respective EDM $d_{\tau}$ is generated by the Glashow-Iliopoulos-Maiani mechanism at very high order in the coupling constant [10]. The error with order of magnitude of $10^{-8}$ is an indication that SM extensions which predict values for $a_{\tau}$ above this level are worth studying. It is also worthwhile to study new mechanisms and new $\tau$ pair production modes in association with a photon $\tau^{+} \tau^{-} \gamma$ at the future $e^{+} e^{-}$linear collider at the $\gamma \gamma$ mode.

The SM predicts $C P$ violation, which is necessary for the existence of the EDM of a variety of physical systems. The EDM provides a direct experimental probe of $C P$ violation [11-13], a feature of the SM and physics BSM. Precise measurement of the EDM of fundamental charged particles provides a significant probe of physics BSM.

The sensitivity to the MM and EDM of the $\tau$-lepton has been studied in both theoretical and experimental contexts, some of which are summarized in Table I. Furthermore, there has been extensive theoretical work done in BSM 
TABLE I. Summary of experimental and theoretical limits on the electromagnetic dipole moments of the $\tau$-lepton.

\begin{tabular}{|c|c|c|c|}
\hline Collaboration & Experimental limit & C.L. & Reference \\
\hline DELPHI & $-0.052<a_{\tau}<0.013$ & $95 \%$ & [42] \\
\hline L3 & $-0.052<a_{\tau}<0.058$ & $95 \%$ & [43] \\
\hline OPAL & $-0.068<a_{\tau}<0.065$ & $95 \%$ & [44] \\
\hline \multirow[t]{2}{*}{ BELLE } & $-2.2<\operatorname{Re}\left(d_{\tau}\left(10^{-17} e \mathrm{~cm}\right)\right)<4.5$ & $95 \%$ & [45] \\
\hline & $-2.5<\operatorname{Im}\left(d_{\tau}\left(10^{-17} e \mathrm{~cm}\right)\right)<0.8$ & $95 \%$ & \\
\hline DELPHI & $-0.22<d_{\tau}\left(10^{-16}\right.$ ecm $)<0.45$ & $95 \%$ & [42] \\
\hline L3 & $\left|\operatorname{Re}\left(d_{\tau}\left(10^{-16} \mathrm{ecm}\right)\right)\right|<3.1$ & $95 \%$ & [43] \\
\hline OPAL & $\left|\operatorname{Re}\left(d_{\tau}\left(10^{-16} e \mathrm{~cm}\right)\right)\right|<3.7$ & $95 \%$ & [44] \\
\hline \multirow[t]{2}{*}{ ARGUS } & $\mid \operatorname{Re}\left(d_{\tau}\left(10^{-16}\right.\right.$ ecm $\left.)\right) \mid<4.6$ & $95 \%$ & [46] \\
\hline & $\left|\operatorname{Im}\left(d_{\tau}\left(10^{-16} e c m\right)\right)\right|<1.8$ & $95 \%$ & \\
\hline Model & Theoretical limit & C.L. & Reference \\
\hline L3 data & $a_{\tau} \leq 0.11$ & $90 \%$ & [47] \\
\hline Electroweak measurements & $-0.004<a_{\tau}<0.006$ & $95 \%$ & [48] \\
\hline LEP1, SLC, LEP2 data & $-0.007<a_{\tau}<0.005$ & $95 \%$ & [49] \\
\hline Total cross section & $a_{\tau}<0.023$ & $95 \%$ & [50] \\
\hline L3 data & $d_{\tau} \leq 6 \times 10^{-16} \mathrm{ecm}$ & $90 \%$ & [47] \\
\hline Electroweak measurements & $d_{\tau} \leq 1.1 \times 10^{-17} \mathrm{ecm}$ & $95 \%$ & [48] \\
\hline Cross section & $d_{\tau} \leq 1.6 \times 10^{-16} \mathrm{ecm}$ & $90 \%$ & [51] \\
\hline
\end{tabular}

models that contributes to dipole moments of charged leptons: $C P$-violation BSM [14], extra dimensions [15], seesaw model [16], version III of the two-higgs-doublet model [17], noncommutative geometry [18], nonuniversal extra dimensions [19], left-right symmetric model [20], $E_{6}$ superstring models [21], simplest little Higgs model [22], and 331 model [23]. There are also bounds independent of the model such as $\gamma p$ collisions [24], $e^{-} \gamma$ scattering [25], and $\gamma \gamma$ collisions $[26,27]$. Other limits on the MM and EDM of the $\tau$-lepton are reported in Refs. [7,28-41].

In this paper, we establish model-independent sensitivity estimates on the dipole moments $a_{\tau}$ and $d_{\tau}$ of the $\tau$-lepton using $\gamma \gamma \rightarrow \tau^{+} \tau^{-}$and $\gamma \gamma \rightarrow \tau^{+} \tau^{-} \gamma$ reactions. An interesting feature of these reactions is that they are extremely clean processes because there is no interference with weak interactions as they are purely quantum electrodynamics (QED) processes. Furthermore, the high center-of-mass energies proposed for the Compact Linear Collider (CLIC) make it an appropriate machine to probe the MM and EDM which are more sensitive to the high energy and high luminosity of the collider.

The CLIC [52-55] is a proposal for a future $e^{+} e^{-}$linear collider at CERN in the High Luminosity-Large Hadron Collider (HL-LHC) era. The machine is designed to make full use of the physics potential of CLIC with an initial operation at center-of-mass energy $\sqrt{s}=380 \mathrm{GeV}$ and luminosity $\mathcal{L}=500 \mathrm{fb}^{-1}$. The $\sqrt{s}=1500 \mathrm{GeV} \quad(\mathcal{L}=$ $\left.1500 \mathrm{fb}^{-1}\right)$ and $\sqrt{s}=3000 \mathrm{GeV}\left(\mathcal{L}=3000 \mathrm{fb}^{-1}\right)$ stages at center-of-mass energies focus on exploring physics BSM. In summary, the CLIC project offers an ample physics program for approximately 20 years, with great discovery potential for new physics. The CLIC can reach scales of up to several tens of $\mathrm{TeV}$ through indirect searches and with very precise measurements.

We consider the following parameters of the CLIC for our study: $\sqrt{s}=380,1500,3000 \mathrm{GeV}, \mathcal{L}=10,50,100,300$, $500,1000,1500,2000,3000 \mathrm{fb}^{-1}$, and we consider systematic uncertainties of $\delta_{\text {sys }}=0 \%, 3 \%, 5 \%$. With these parameters as input, we established model-independent sensitivity estimates on the electromagnetic dipole moments of the $\tau$-lepton at $95 \%$ C.L. We obtain strong sensitivity in comparison to the bounds given by the DELPHI, L3, OPAL, BELLE, and ARGUS Collaborations [42-46] (see Table I).

The remainder of this paper is organized as follows: In Sec. II, we study the total cross section and the electromagnetic dipole moments of the $\tau$-lepton through the $\gamma \gamma \rightarrow$ $\tau^{+} \tau^{-}$and $\gamma \gamma \rightarrow \tau^{+} \tau^{-} \gamma$ reactions. In Sec. III, we give our conclusions.

\section{TWO-PHOTON PROCESSES $\gamma \gamma \rightarrow \tau^{+} \tau^{-}$AND $\gamma \gamma \rightarrow \tau^{+} \tau^{-} \gamma$}

We take advantage of our previous works on the collision modes $\gamma \gamma, \gamma \gamma^{*}$, and $\gamma^{*} \gamma^{*}[26,56-58]$ to calculate the total cross section for the $\gamma \gamma \rightarrow \tau^{+} \tau^{-}$and $\gamma \gamma \rightarrow \tau^{+} \tau^{-} \gamma$ reactions. The corresponding Feynman diagrams for these processes are given in Figs. 1 and 2, respectively.

We deduce sensitivity estimates on the electromagnetic dipole moments of the $\tau$-lepton $a_{\tau}$ and $d_{\tau}$ via the twophoton processes $\gamma \gamma \rightarrow \tau^{+} \tau^{-}$and $\gamma \gamma \rightarrow \tau^{+} \tau^{-} \gamma$. These processes are of interest for a number of reasons. First, they are sensitive to the $a_{\tau}$ and $d_{\tau}$. Additionally, increased cross sections for high energies and the absence or strong suppression of weak contributions are complementary 


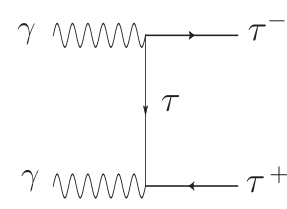

(1)

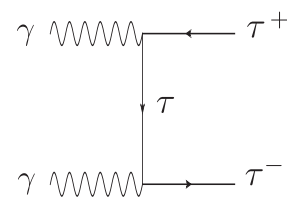

(2)
FIG. 1. The Feynman diagrams for the process $\gamma \gamma \rightarrow \tau^{+} \tau^{-}$.

aspects of the two-photon processes in contrast to the direct processes $e^{+} e^{-} \rightarrow \tau^{+} \tau^{-}$[46,51], $e^{+} e^{-} \rightarrow \tau^{+} \tau^{-} \gamma$ [43], and $Z \rightarrow \tau^{+} \tau^{-} \gamma[44,47]$. Another important point is the availability of high luminosity photon beams due to bremsstrahlung as a by-product in planned high energy colliders. Finally, the future CLIC can produce very hard photons at high luminosity in Compton backscattering of laser light off high energy $e^{+} e^{-}$beams.

In order to determine the sensitivity on the MM and EDM of the $\tau$-lepton, we calculate the total cross section of the reactions $\gamma \gamma \rightarrow \tau^{+} \tau^{-}$and $\gamma \gamma \rightarrow \tau^{+} \tau^{-} \gamma$. The most general parametrization for the electromagnetic current between on-shell $\tau$-lepton and the photon is given by $[7,47,48,59]$

$$
\begin{aligned}
\Gamma_{\tau}^{\alpha}= & e F_{1}\left(q^{2}\right) \gamma^{\alpha}+\frac{i e}{2 m_{\tau}} F_{2}\left(q^{2}\right) \sigma^{\alpha \mu} q_{\mu}+\frac{e}{2 m_{\tau}} F_{3}\left(q^{2}\right) \sigma^{\alpha \mu} q_{\mu} \gamma_{5} \\
& +e F_{4}\left(q^{2}\right) \gamma_{5}\left(\gamma^{\alpha}-\frac{2 q^{\alpha} m_{\tau}}{q^{2}}\right)
\end{aligned}
$$

where $e$ is the charge of the electron, $m_{\tau}$ is the mass of the $\tau$-lepton, $\sigma^{\alpha \mu}=\frac{i}{2}\left[\gamma^{\alpha}, \gamma^{\mu}\right]$ represents the spin $1 / 2$ angular momentum tensor, and $q=p^{\prime}-p$ is the momentum transfer. In the static (classical) limit, the $q^{2}$-dependent form factors $F_{1,2,3,4}\left(q^{2}\right)$ have familiar interpretations for

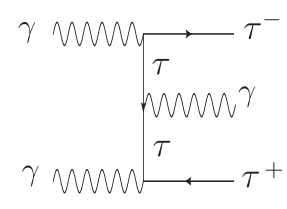

(1)

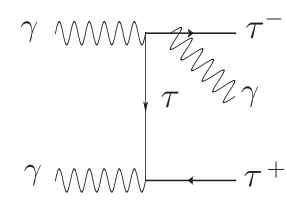

(3)

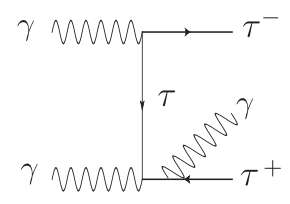

$(5)$

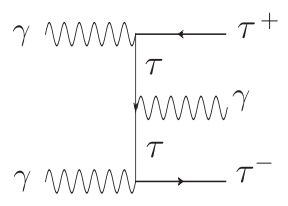

(2)

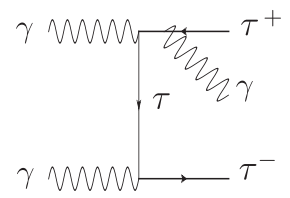

(4)

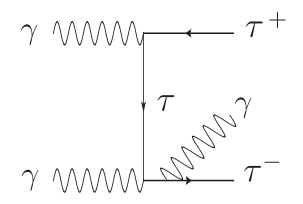

(6)
FIG. 2. The Feynman diagrams for the process $\gamma \gamma \rightarrow \tau^{+} \tau^{-} \gamma$. $q^{2}=0: F_{1}(0)=Q_{\tau}$ is the electric charge; $F_{2}(0)=a_{\tau}$ is the anomalous MM and $F_{3}(0)=\frac{2 m_{\tau}}{e} d_{\tau}$ with $d_{\tau}$ the EDM. $F_{4}\left(q^{2}\right)$ is the anapole form factor.

In phenomenological and experimental searches, most of the $\tau$-lepton electromagnetic vertices search involve offshell $\tau$-leptons. Indeed in these studies, one of the $\tau$-lepton is off-shell and measured quantity is not directly $a_{\tau}$ and $d_{\tau}$. Deviations of the $\tau$-lepton dipole moments from the SM values are thus examined using an effective Lagrangian approach. It is common to study new physics in a modelindependent way through the effective Lagrangian approach which is defined by high-dimensional operators which lead to anomalous $\tau^{+} \tau^{-} \gamma$ coupling. The operators corresponding to charge radius and the anapole moment do not concern us here. In this study, we apply the dimensionsix effective operators that contribute to the electric and magnetic dipole moments of the $\tau$-lepton at the tree level given by Refs. [60-62]:

$$
L_{\mathrm{eff}}=\frac{1}{\Lambda^{2}}\left[C_{L W}^{33} Q_{L W}^{33}+C_{L B}^{33} Q_{L B}^{33}+\text { H.c. }\right],
$$

where

$$
\begin{gathered}
Q_{L W}^{33}=\left(\bar{\ell}_{\tau} \sigma^{\mu \nu} \tau_{R}\right) \sigma^{I} \varphi W_{\mu \nu}^{I}, \\
Q_{L B}^{33}=\left(\bar{\ell}_{\tau} \sigma^{\mu \nu} \tau_{R}\right) \varphi B_{\mu \nu} .
\end{gathered}
$$

Here, $\varphi$ and $\ell_{\tau}$ are the Higgs and the left-handed $S U(2)$ doublets, $\sigma^{I}$ are the Pauli matrices, and $W_{\mu \nu}^{I}$ and $B_{\mu \nu}$ are the gauge field strength tensors.

After the electroweak symmetry breaking from the effective Lagrangian in Eq. (5), the $C P$ even $\kappa$ and $C P$ odd $\tilde{\kappa}$ parameters are obtained,

$$
\begin{aligned}
& \kappa=\frac{2 m_{\tau}}{e} \frac{\sqrt{2} v}{\Lambda^{2}} \operatorname{Re}\left[\cos \theta_{W} C_{L B}^{33}-\sin \theta_{W} C_{L W}^{33}\right], \\
& \tilde{\kappa}=\frac{2 m_{\tau}}{e} \frac{\sqrt{2} v}{\Lambda^{2}} \operatorname{Im}\left[\cos \theta_{W} C_{L B}^{33}-\sin \theta_{W} C_{L W}^{33}\right],
\end{aligned}
$$

where $v=246 \mathrm{GeV}$ and $\sin \theta_{W}$ is the weak mixing angle.

These parameters are related to the contribution of the anomalous magnetic and electric dipole moments of the $\tau$-lepton through the following relations:

$$
\begin{gathered}
\kappa=\tilde{a}_{\tau}, \\
\tilde{\kappa}=\frac{2 m_{\tau}}{e} \tilde{d}_{\tau} .
\end{gathered}
$$

\section{A. $\gamma \gamma \rightarrow \tau^{+} \tau^{-}$cross section}

All signal cross sections in this paper are computed using the CALCHEP 3.6.30 package [63], which can computate the Feynman diagrams, integrate over multiparticle phase space, and simulate events. In addition, we consider the following basic acceptance cuts for $\tau^{+} \tau^{-}$events at the CLIC: 


$$
\begin{aligned}
p_{t}^{\tau, \bar{\tau}} & >20 \mathrm{GeV}, \\
\left|\eta^{\tau, \bar{\tau}}\right| & <2.5, \\
\Delta R(\tau, \bar{\tau}) & >0.4 .
\end{aligned}
$$

We apply these cuts to reduce the background and to optimize the signal sensitivity. In Eq. (12), $p_{t}^{\tau, \bar{\tau}}$ is the transverse momentum of the final state particles, $\eta^{\tau, \bar{\tau}}$ is the pseudorapidity which reduces the contamination from other particles misidentified as $\tau$, and $\Delta R(\tau, \bar{\tau})$ is the separation of the final state particles.

For Fig. 1, the square matrix elements for the process $\gamma \gamma \rightarrow \tau^{+} \tau^{-}$(for $M_{1}$ and $M_{2}$, respectively) as a function of the Mandelstam invariants $\hat{s}, \hat{t}$, and $\hat{u}$ are given by

$$
\begin{aligned}
\left|M_{1}\right|^{2}= & \frac{16 \pi^{2} Q_{\tau}^{2} \alpha_{e}^{2}}{2 m_{\tau}^{4}\left(\hat{t}-m_{\tau}^{2}\right)^{2}}\left[48 \kappa\left(m_{\tau}^{2}-\hat{t}\right)\left(m_{\tau}^{2}+\hat{s}-\hat{t}\right) m_{\tau}^{4}-16\left(3 m_{\tau}^{4}-m_{\tau}^{2} \hat{s}+\hat{t}(\hat{s}+\hat{t})\right) m_{\tau}^{4}+2\left(m_{\tau}^{2}-\hat{t}\right)\left(\kappa ^ { 2 } \left(17 m_{\tau}^{4}\right.\right.\right. \\
& \left.\left.+(22 \hat{s}-26 \hat{t}) m_{\tau}^{2}+\hat{t}(9 \hat{t}-4 \hat{s})\right)+\tilde{\kappa}^{2}\left(17 m_{\tau}^{2}+4 \hat{s}-9 \hat{t}\right)\left(m_{\tau}^{2}-\hat{t}\right)\right) m_{\tau}^{2}+12 \kappa\left(\kappa^{2}+\tilde{\kappa}^{2}\right) \hat{s}\left(m_{\tau}^{3}-m_{\tau} \hat{t}\right)^{2} \\
& \left.-\left(\kappa^{2}+\tilde{\kappa}^{2}\right)^{2}\left(m_{\tau}^{2}-\hat{t}\right)^{3}\left(m_{\tau}^{2}-\hat{s}-\hat{t}\right)\right], \\
\left|M_{2}\right|^{2}= & \frac{-16 \pi^{2} Q_{\tau}^{2} \alpha_{e}^{2}}{2 m_{\tau}^{4}\left(\hat{u}-m_{\tau}^{2}\right)^{2}}\left[48 \kappa\left(m_{\tau}^{4}+(\hat{s}-2 \hat{t}) m_{\tau}^{2}+\hat{t}(\hat{s}+\hat{t})\right) m_{\tau}^{4}+16\left(7 m_{\tau}^{4}-(3 \hat{s}+4 \hat{t}) m_{\tau}^{2}+\hat{t}(\hat{s}+\hat{t})\right) m_{\tau}^{4}\right. \\
& +2\left(m_{\tau}^{2}-\hat{t}\right)\left(\kappa^{2}\left(m_{\tau}^{4}+(17 \hat{s}-10 \hat{t}) m_{\tau}^{2}+9 \hat{t}(\hat{s}+\hat{t})\right)+\tilde{\kappa}^{2}\left(m_{\tau}^{2}-9 \hat{t}\right)\left(m_{\tau}^{2}-\hat{t}-\hat{s}\right)\right) m_{\tau}^{2} \\
& \left.+\left(\kappa^{2}+\tilde{\kappa}^{2}\right)^{2}\left(m_{\tau}^{2}-\hat{t}\right)^{3}\left(m_{\tau}^{2}-\hat{s}-\hat{t}\right)\right], \\
& \\
& \left.\quad+\left(\kappa^{2}\left(16 m_{\tau}^{6}-m_{\tau}^{4}(15 \hat{s}+32 \hat{t})+m_{\tau}^{2}(15 \hat{s})^{2}+14 \hat{t} \hat{s}+16 \hat{t}\right)^{2}\right)+\hat{s} \hat{t}(\hat{s}+\hat{t})\right) \\
& \left.\left.\left.\quad+\tilde{\kappa}^{2}\left(16 m_{\tau}^{6}-m_{\tau}^{4}(15 \hat{s}+32 \hat{t})+m_{\tau}^{2}(5 \hat{s})^{2}+14 \hat{t} \hat{s}+16 \hat{t}\right)^{2}\right)+\hat{s} \hat{t}(\hat{s}+\hat{t})\right)\right)-4 \kappa \hat{s}\left(\kappa^{2}+\tilde{\kappa}^{2}\right) \\
& \left.\quad \times\left(m_{\tau}^{4}+m_{\tau}^{2}(\hat{s}-2 \hat{t})+\hat{t}(\hat{s}+\hat{t})\right)-2 \hat{s}\left(\kappa^{2}+\tilde{\kappa}^{2}\right)^{2}\left(m_{\tau}^{4}-2 \hat{t} m_{\tau}^{2}+\hat{t}(\hat{s}+\hat{t})\right)\right],
\end{aligned}
$$

where $\hat{s}=\left(p_{1}+p_{2}\right)^{2}=\left(p_{3}+p_{4}\right)^{2}, \quad \hat{t}=\left(p_{1}-p_{3}\right)^{2}=$ $\left(p_{4}-p_{2}\right)^{2}, \hat{u}=\left(p_{3}-p_{2}\right)^{2}=\left(p_{1}-p_{4}\right)^{2}$, and $p_{1}$ and $p_{2}$ are the four-momentum of the incoming photons, $p_{3}$ and $p_{4}$ are the momentum of the outgoing $\tau$-lepton, $Q_{\tau}$ is the $\tau$-lepton charge, $\alpha_{e}$ is the fine-structure constant, and $m_{\tau}$ is the mass of the $\tau$.

The most promising mechanism to generate energetic photon beams in a linear collider is Compton backscattering. Compton backscattered photons interact with each other and generate the process $\gamma \gamma \rightarrow \tau^{+} \tau^{-}$. The spectrum of Compton backscattered photons $[64,65]$ is given by

$f_{\gamma}(y)=\frac{1}{g(\zeta)}\left[1-y+\frac{1}{1-y}-\frac{4 y}{\zeta(1-y)}+\frac{4 y^{2}}{\zeta^{2}(1-y)^{2}}\right]$,

where

$g(\zeta)=\left(1-\frac{4}{\zeta}-\frac{8}{\zeta^{2}}\right) \log (\zeta+1)+\frac{1}{2}+\frac{8}{\zeta}-\frac{1}{2(\zeta+1)^{2}}$, with

$$
y=\frac{E_{\gamma}}{E_{e}}, \quad \zeta=\frac{4 E_{0} E_{e}}{M_{e}^{2}}, \quad y_{\max }=\frac{\zeta}{1+\zeta} .
$$

Here, $E_{0}$ and $E_{e}$ are energy of the incoming laser photon and initial energy of the electron beam before Compton backscattering, and $E_{\gamma}$ is the energy of the backscattered photon. The maximum value of $y$ reaches 0.83 when $\zeta=4.8$.

The total cross section is given by

$$
\sigma=\int f_{\gamma}(x) f_{\gamma}(x) d \hat{\sigma} d E_{1} d E_{2}
$$

Next, we present the total cross section as a polynomial in powers of $\kappa(\tilde{\kappa})$. This provides more precise and convenient information for the study of the process $\gamma \gamma \rightarrow \tau^{+} \tau^{-}$. We consider the following cases: 
(i) For $\sqrt{s}=380 \mathrm{GeV}$,

$$
\begin{aligned}
\sigma(\kappa, \tilde{\kappa})= & {\left[\left(9.73 \times 10^{6}\right) \kappa^{4}+\left(9.73 \times 10^{6}\right) \tilde{\kappa}^{4}+\left(1.97 \times 10^{7}\right) \kappa^{2} \tilde{\kappa}^{2}+\left(8.22 \times 10^{4}\right) \kappa \tilde{\kappa}^{2}\right.} \\
& \left.+\left(8.18 \times 10^{4}\right) \kappa^{3}+\left(8.13 \times 10^{4}\right) \kappa^{2}+\left(8.26 \times 10^{4}\right) \tilde{\kappa}^{2}+\left(1.11 \times 10^{2}\right) \kappa+38.75\right](\mathrm{pb}) .
\end{aligned}
$$

(ii) For $\sqrt{s}=1500 \mathrm{GeV}$,

$$
\begin{aligned}
\sigma(\kappa, \tilde{\kappa})= & {\left[\left(1.54 \times 10^{8}\right) \kappa^{4}+\left(1.54 \times 10^{8}\right) \tilde{\kappa}^{4}+\left(3.08 \times 10^{8}\right) \kappa^{2} \tilde{\kappa}^{2}+\left(8.41 \times 10^{4}\right) \kappa \tilde{\kappa}^{2}\right.} \\
& \left.+\left(8.42 \times 10^{8}\right) \kappa^{3}+\left(8.80 \times 10^{4}\right) \kappa^{2}+\left(8.81 \times 10^{4}\right) \tilde{\kappa}^{2}+(17.5) \kappa+6.00\right](\mathrm{pb}) .
\end{aligned}
$$

(iii) For $\sqrt{s}=3000 \mathrm{GeV}$,

$$
\begin{aligned}
\sigma(\kappa, \tilde{\kappa})= & {\left[\left(6.17 \times 10^{8}\right) \kappa^{4}+\left(6.17 \times 10^{8}\right) \tilde{\kappa}^{4}+\left(1.23 \times 10^{9}\right) \kappa^{2} \tilde{\kappa}^{2}+\left(8.64 \times 10^{4}\right) \kappa \tilde{\kappa}^{2}\right.} \\
& \left.+\left(9.13 \times 10^{4}\right) \kappa^{3}+\left(8.72 \times 10^{4}\right) \kappa^{2}+\left(8.83 \times 10^{4}\right) \tilde{\kappa}^{2}-(1.21) \kappa+1.97\right](\mathrm{pb})
\end{aligned}
$$

As seen in Eqs. (20)-(22), the linear, quadratic, and cubic terms in $\kappa(\tilde{\kappa})$ arise from the interference between SM and anomalous amplitudes, whereas the quartic terms are purely anomalous. The term independent of $\kappa(\tilde{\kappa})$ corresponds to the cross section at $\kappa=\tilde{\kappa}=0$ and represents the contribution of the SM cross section. It is worth mentioning that the spin averaged cross section brings even powers of $\tilde{\kappa}$ in the result and that means $C P$ is even. $C P$ odd terms appear if the spin dependent cross section is taken into account. However, in this work, spin dependent calculation is out of scope. For this reason, the magnitudes of negative and positive parts of the limits on $d_{\tau}$ are the same. Similar reasoning can be given for the process $\gamma \gamma \rightarrow \tau^{+} \tau^{-} \gamma$.

\section{B. Sensitivity on the $\tilde{\boldsymbol{a}}_{\tau}$ and $\tilde{\boldsymbol{d}}_{\tau}$ through $\gamma \gamma \rightarrow \tau^{+} \tau^{-}$at the CLIC}

For our numerical analysis of the total cross section $\sigma_{N P}\left(\gamma \gamma \rightarrow \tau^{+} \tau^{-}\right)=\sigma_{N P}(\sqrt{s}, \kappa, \tilde{\kappa})$, and of the electromagnetic dipole moments of the $\tau$-lepton, the free parameters are the center-of-mass energy $\sqrt{s}$, the integrated luminosity $\mathcal{L}$ of the CLIC, and the factors $\kappa$ and $\tilde{\kappa}$. We also consider the acceptance cuts given in Eq. (12). In addition, we take into account the systematic uncertainties for the collider and use the usual formula for the $\chi^{2}$ function $[24-26,66]$,

$$
\chi^{2}=\left(\frac{\sigma_{\mathrm{SM}}-\sigma_{N P}(\sqrt{s}, \kappa, \tilde{\kappa})}{\sigma_{\mathrm{SM}} \delta}\right)^{2}
$$

where $\sigma_{N P}(\sqrt{s}, \kappa, \tilde{\kappa})$ is the total cross section including contributions from the SM and new physics, $\delta=\sqrt{\left(\delta_{\mathrm{st}}\right)^{2}+\left(\delta_{\mathrm{sys}}\right)^{2}}, \delta_{\mathrm{st}}=\frac{1}{\sqrt{N_{\mathrm{SM}}}}$ is the statistical error, $\delta_{\text {sys }}$ is the systematic error, and $N_{\mathrm{SM}}$ is the number of signal expected events, $N_{\mathrm{SM}}=\mathcal{L}_{\text {int }} \times B R \times \sigma_{\mathrm{SM}}$, where $\mathcal{L}_{\text {int }}$ is the integrated CLIC luminosity. Furthermore, since the $\tau$-lepton decays roughly $35 \%$ of the time leptonically and $65 \%$ of the time to one or more hadrons, we consider one of the $\tau$-leptons decays leptonically and the other hadronically to obtain the signal. We then assume the branching ratio of the two- $\tau$ in the final state to be $\mathrm{BR}=0.46$. The main $\tau$-decay branching ratios are given in Refs. $[6,67,68]$.

Systematic uncertainties may occur when identifying the $\tau$-lepton due to many factors. Although we do not have any CLIC reports [69-71] to know exactly what the systematic uncertainties are for our processes, we can assume some of their general values. The DELPHI Collaboration examined the anomalous magnetic and electric dipole moments of the $\tau$-lepton through the process $e^{+} e^{-} \rightarrow e^{+} e^{-} \tau^{+} \tau^{-}$during the years 1997-2000 at collision energy $\sqrt{s}$ between 183 and $208 \mathrm{GeV}$ [42]. Relative systematic cross-section errors of the process $e^{+} e^{-} \rightarrow e^{+} e^{-} \tau^{+} \tau^{-}$are given in Table II. The process $e^{+} e^{-} \rightarrow e^{+} e^{-} \tau^{+} \tau^{-}$was also studied with the L3 detector for center-of-mass energies $161 \mathrm{GeV} \leq \sqrt{s} \leq$ $209 \mathrm{GeV}$ at LEP [43]. The anomalous magnetic and electric dipole moments of the $\tau$-lepton via the process $p p \rightarrow p p \tau^{+} \tau^{-}$with $2 \%$ of the total systematic uncertainties at the LHC were investigated phenomenologically in Ref. [72]. Work in this regard is done by ATLAS and CMS Collaborations [73,74]. The $\tau$ tagging efficiencies were also studied using the International Large Detector (ILD) [75], a proposed detector concept for the International Linear

TABLE II. Systematic errors given by the DELPHI Collaboration [42].

\begin{tabular}{lcccc}
\hline \hline & 1997 & 1998 & 1999 & 2000 \\
\hline Trigger efficiency & 7.0 & 2.7 & 3.6 & 4.5 \\
Selection efficiency & 5.1 & 3.2 & 3.0 & 3.0 \\
Background & 1.7 & 0.9 & 0.9 & 0.9 \\
Luminosity & 0.6 & 0.6 & 0.6 & 0.6 \\
Total & 8.9 & 4.3 & 4.7 & 5.4 \\
\hline \hline
\end{tabular}




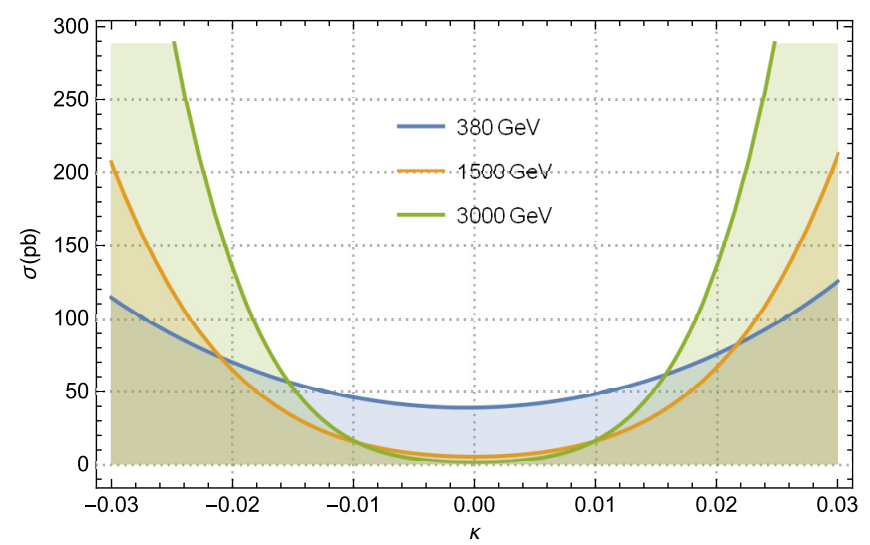

FIG. 3. The total cross sections of the process $\gamma \gamma \rightarrow \tau^{+} \tau^{-}$as a function of $\kappa$ for center-of-mass energies of $\sqrt{s}=380,1500$, $3000 \mathrm{GeV}$.

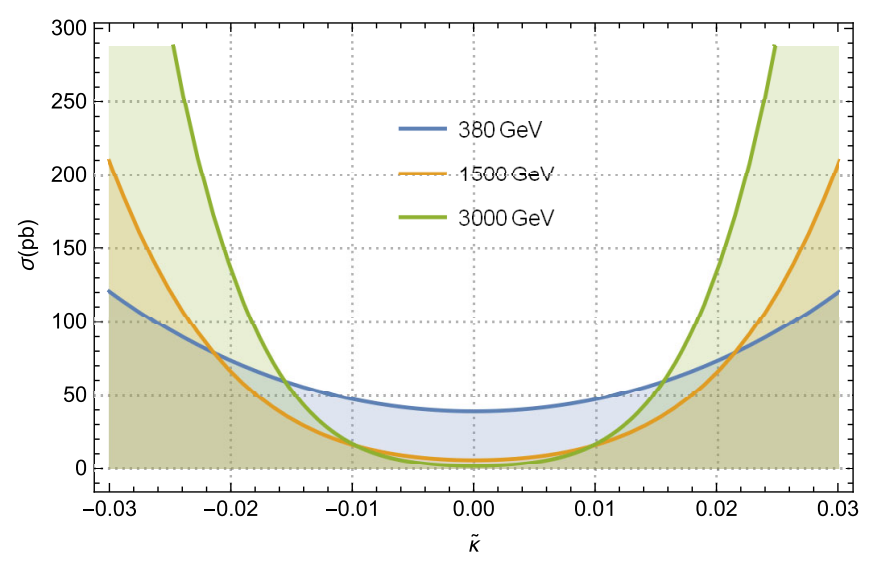

FIG. 4. Same as in Fig. 3, but for $\tilde{\kappa}$.

Collider (ILC). Because of these difficulties, $\tau$ identification efficiencies are always calculated for specific processes, luminosity, and kinematic parameters. These studies are currently being carried out by various groups for selected productions. For realistic efficiency, we need a detailed study for our specific process and kinematic parameters. For all of these reasons, kinematic cuts contain

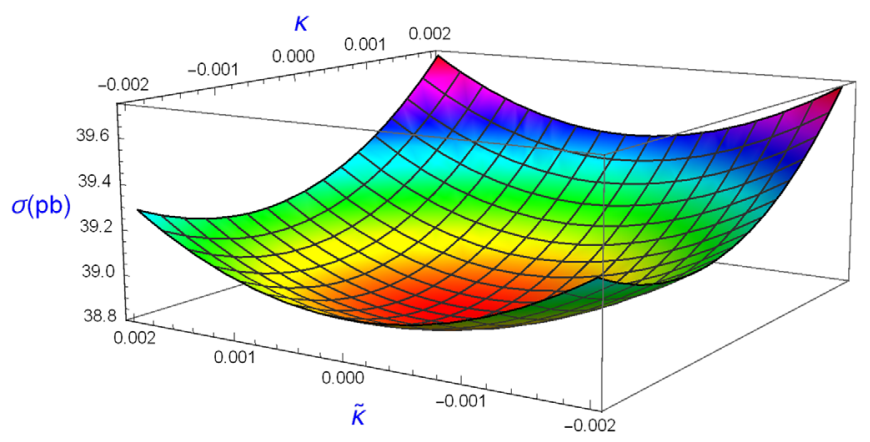

FIG. 5. The total cross sections of the process $\gamma \gamma \rightarrow \tau^{+} \tau^{-}$as a function of $\kappa$ and $\tilde{\kappa}$ for center-of-mass energy of $\sqrt{s}=380 \mathrm{GeV}$.

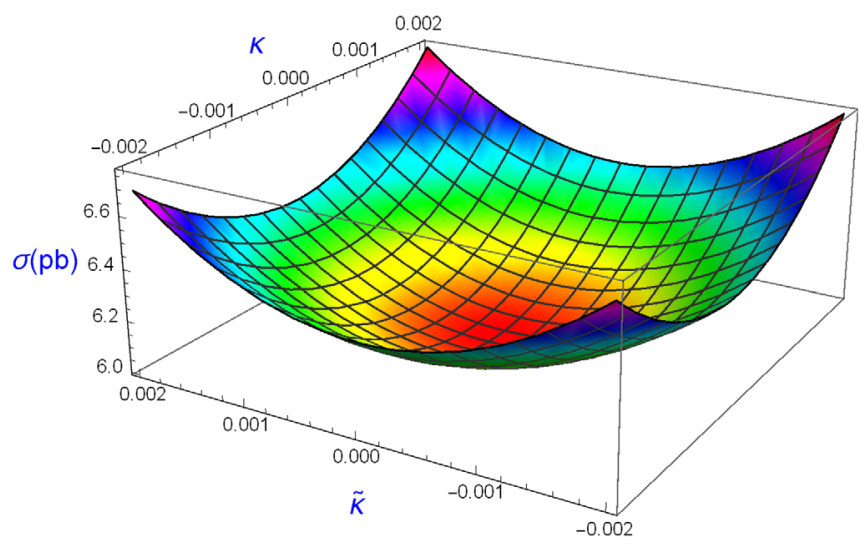

FIG. 6. Same as in Fig. 5, but for $\sqrt{s}=1500 \mathrm{GeV}$.

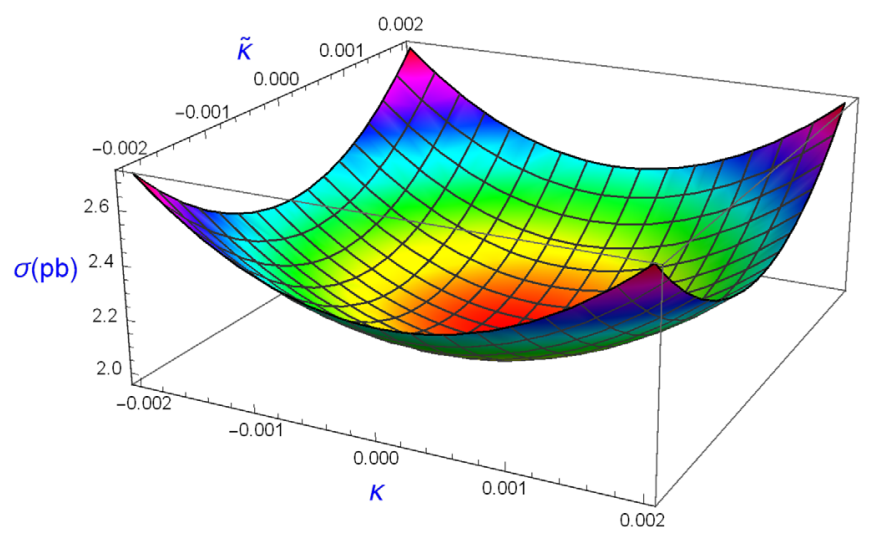

FIG. 7. Same as in Fig. 5, but for $\sqrt{s}=3000 \mathrm{GeV}$.

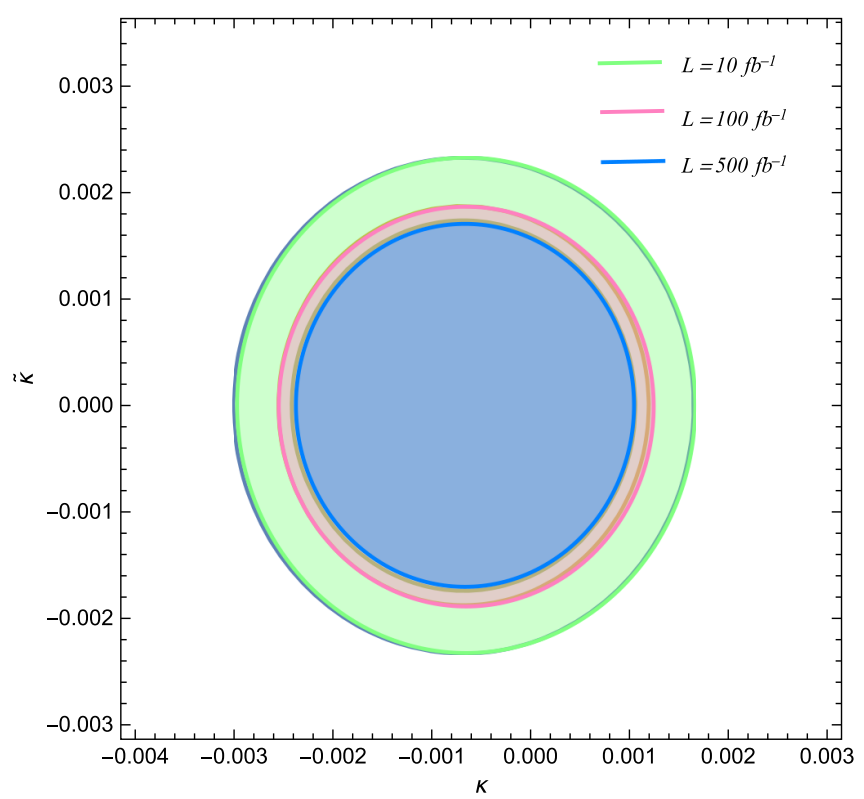

FIG. 8. Sensitivity contours at the $95 \%$ C.L. in the $(\kappa-\tilde{\kappa})$ plane for the process $\gamma \gamma \rightarrow \tau^{+} \tau^{-}$with the $\mathcal{L}=10,100,500 \mathrm{fb}^{-1}$ and for center-of-mass energy of $\sqrt{s}=380 \mathrm{GeV}$. 


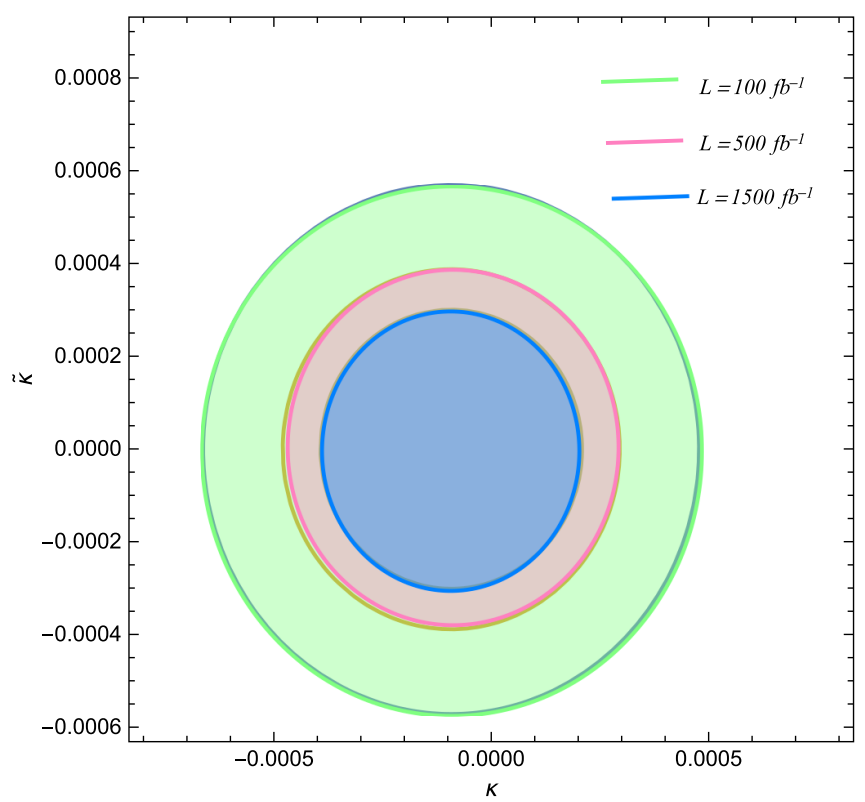

FIG. 9. Same as in Fig. 8, but for $\mathcal{L}=100,500,1500 \mathrm{fb}^{-1}$ and for center-of-mass energy of $\sqrt{s}=1500 \mathrm{GeV}$.

some general values chosen by lepton identification detectors, and efficiency is therefore considered within systematic errors. Taking previous studies into consideration, 3\% and $5 \%$ of total systematic uncertainties were used in our study. It may be assumed that this accelerator will be built in the coming years and the systematic uncertainties will be lower as detector technology develops in the future.

The total cross section $\sigma_{\gamma \gamma \rightarrow \tau^{+} \tau^{-}}(\sqrt{s}, \kappa, \tilde{\kappa})$ is presented as a function of the anomalous couplings $\kappa$ in Fig. 3 and $\tilde{\kappa}$ in Fig. 4 with the center-of-mass energies $\sqrt{s}=380,1500$,

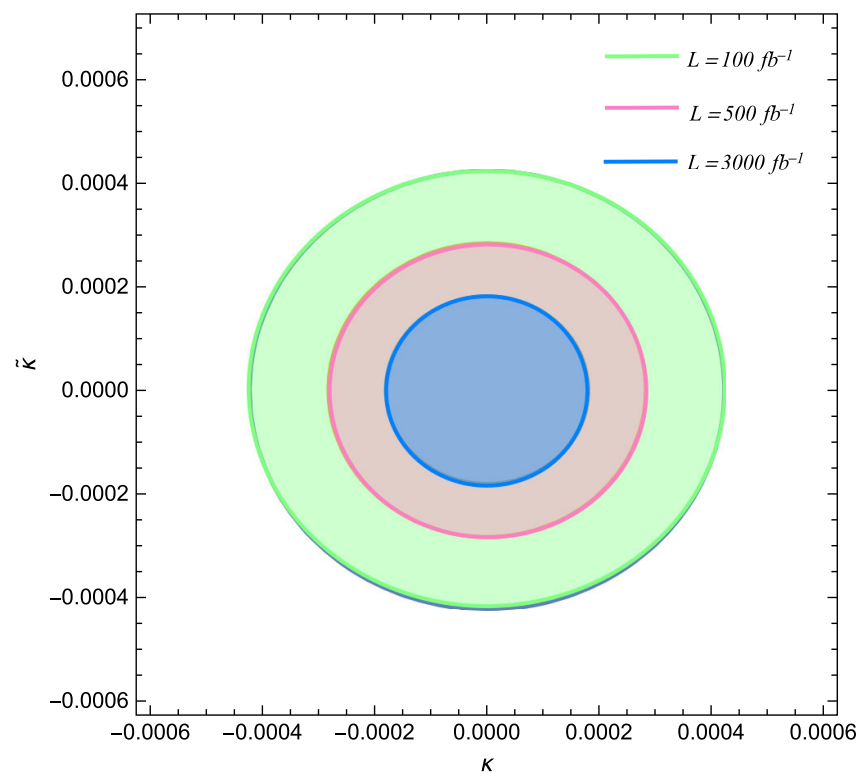

FIG. 10. Same as in Fig. 8, but for $\mathcal{L}=100,500,3000 \mathrm{fb}^{-1}$ and for center-of-mass energy of $\sqrt{s}=3000 \mathrm{GeV}$.

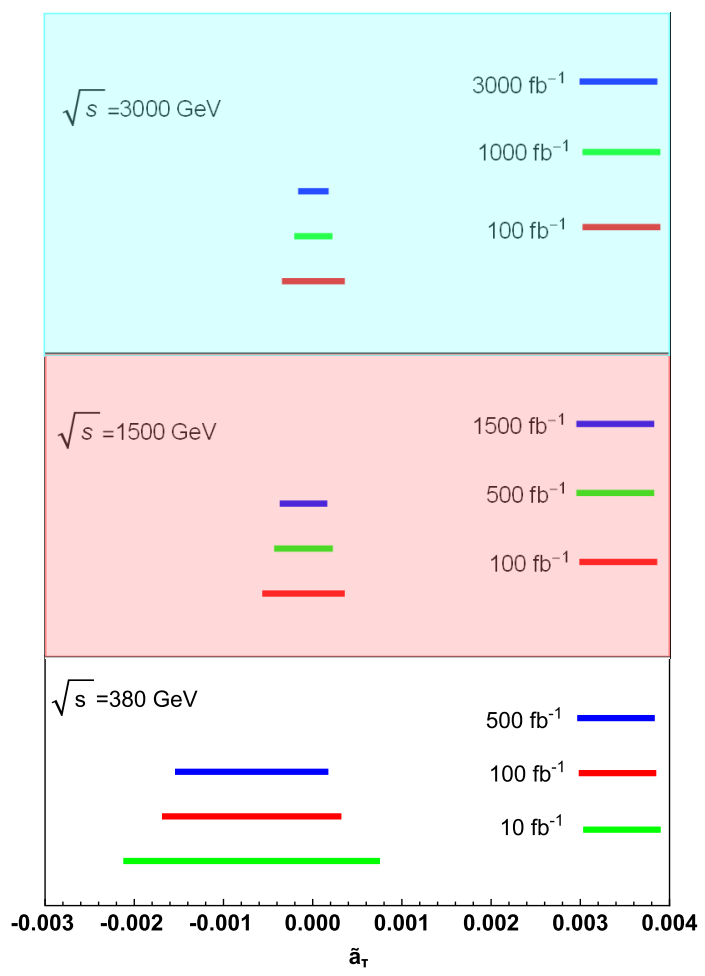

FIG. 11. Comparison of precisions on $\tilde{a}_{\tau}$ in the process $\gamma \gamma \rightarrow$ $\tau^{+} \tau^{-}$expected at the CLIC. We assume luminosities as follows: Top panel: $\mathcal{L}=100,1000,3000 \mathrm{fb}^{-1}$ and $\sqrt{s}=3000 \mathrm{GeV}$. Central panel: $\mathcal{L}=100,500,1500 \mathrm{fb}^{-1}$ and $\sqrt{s}=1500 \mathrm{GeV}$. Bottom panel: $\mathcal{L}=10,100,500 \mathrm{fb}^{-1}$ and $\sqrt{s}=380 \mathrm{GeV}$.

$3000 \mathrm{GeV}$, respectively. The total cross section clearly shows a strong dependence on the anomalous parameters $\kappa$, $\tilde{\kappa}$, and the center-of-mass energy of the collider $\sqrt{s}$. Additionally, the $\sigma_{\gamma \gamma \rightarrow \tau^{+} \tau^{-}}(\sqrt{s}, \kappa, \tilde{\kappa})$ as a function of $\kappa$

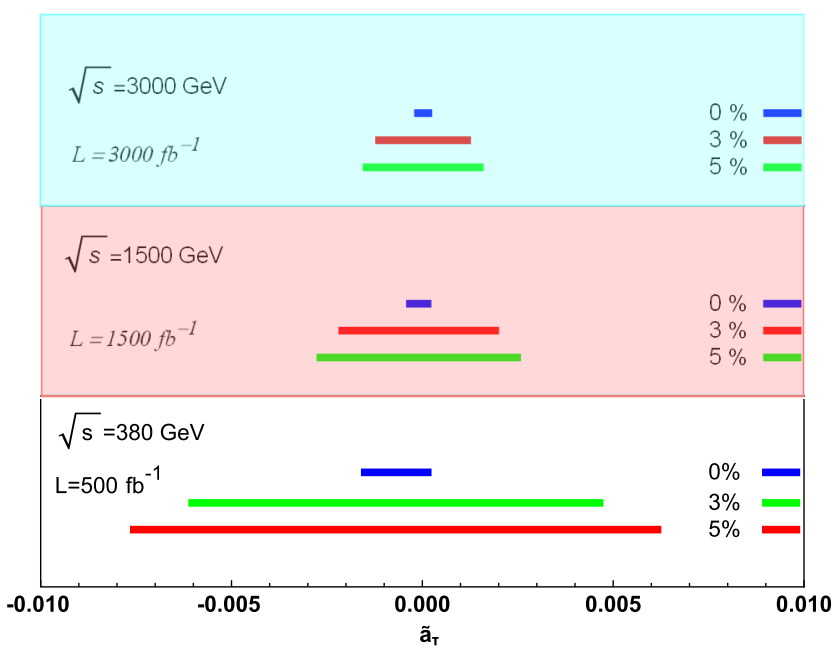

FIG. 12. Same as in Fig. 11, but as follows: Top panel: $\delta_{\text {sys }}=0 \%, 3 \%, 5 \%$ with $\mathcal{L}=3000 \mathrm{fb}^{-1}$ and $\sqrt{s}=3000 \mathrm{GeV}$. Central panel: $\delta_{\text {sys }}=0 \%, 3 \%, 5 \%$ with $\mathcal{L}=1500 \mathrm{fb}^{-1}$ and $\sqrt{s}=1500 \mathrm{GeV}$. Bottom panel: $\delta_{\text {sys }}=0 \%, 3 \%, 5 \%$ with $\mathcal{L}=$ $500 \mathrm{fb}^{-1}$ and $\sqrt{s}=380 \mathrm{GeV}$. 


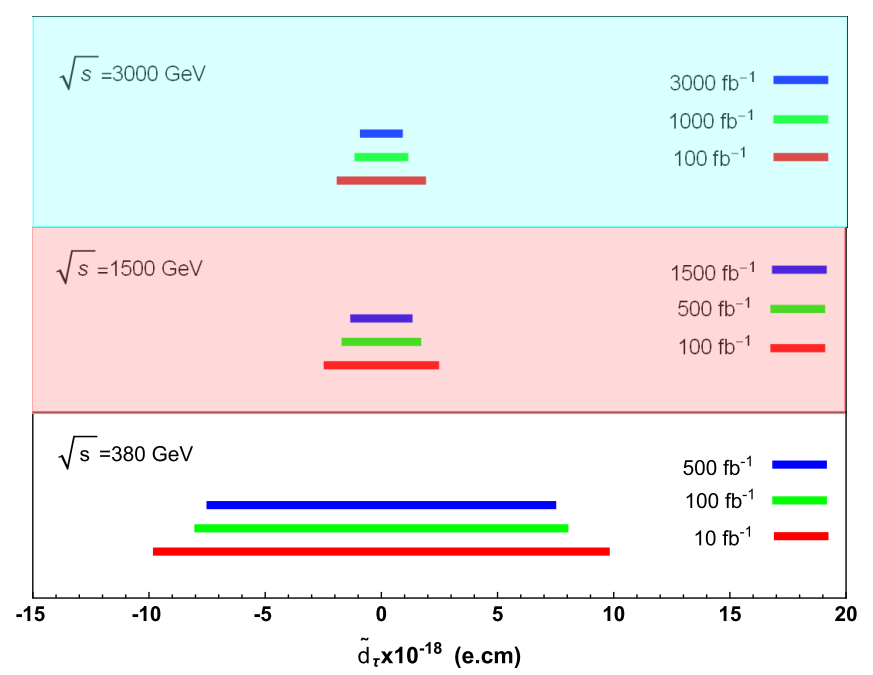

FIG. 13. Same as in Fig. 11, but for $\tilde{d}_{\tau}$.

and $\tilde{\kappa}$ is shown in Figs. 5-7. The surfaces are increased for the lower and upper limits of the parameters $\kappa$ and $\tilde{\kappa}$, showing a strong dependence on these parameters.

Figures $8-10$ show allowed regions at $95 \%$ C.L. in the plane $(\kappa-\tilde{\kappa})$ for the process $\gamma \gamma \rightarrow \tau^{+} \tau^{-}$during the first, second, and third stages of operation of the CLIC, where assumed fixed center-of-mass energies are $\sqrt{s}=380$, $1500,3000 \mathrm{GeV}$ with luminosities $\mathcal{L}=10, \quad 100$, $500 \mathrm{fb}^{-1}, \mathcal{L}=100,500,1500 \mathrm{fb}^{-1}$, and $\mathcal{L}=100,500$, $3000 \mathrm{fb}^{-1}$, respectively, and systematic uncertainties of $\delta_{\text {sys }}=0 \%, 3 \%, 5 \%[42,76]$. See Refs. [72,77] for a more detailed description of the uncertainties.
The achievable precision in the determination of the anomalous magnetic moment $\tilde{a}_{\tau}$ and the electric dipole moment $\tilde{d}_{\tau}$ are summarized in Figs. 11-14 and Tables III-V. These are compared with experimental results of earlier studies for a linear collider as published by the BELLE, DELPHI, L3, and OPAL Collaborations [42-46]. Our results for the twophoton process $\gamma \gamma \rightarrow \tau^{+} \tau^{-}$at the CLIC could improve the sensitivity on anomalous electromagnetic dipole moments of $\tau$-lepton with respect to the existing experimental bounds (see Table I) by 2 orders of magnitude. The best sensitivities obtained on $\tilde{a}_{\tau}$ and $\tilde{d}_{\tau}$ were $-0.00015 \leq \tilde{a}_{\tau} \leq 0.00017$ and $\left|\tilde{d}_{\tau}(\mathrm{ecm})\right|=9.040 \times 10^{-19}$, respectively, as shown in Tables III-V.

We now consider how realistic our assumptions are regarding values of the error parameter delta. The uncertainty that may occur in the error delta value can be caused by the $\mathrm{SM}$ cross section. It should be mentioned that there is no SM calculation at the loop level for the processes $\gamma \gamma \rightarrow \tau^{+} \tau^{-}$ and $\gamma \gamma \rightarrow \tau^{+} \tau^{-} \gamma$ that have been studied in the literature. However, it is expected that the loop contributions are relatively small compared to the SM cross section at the tree level [7,61]. It is important to determine how much the SM cross section differs from our results due to the calculation or the contribution from the loop level, and how this difference may affect our results. We assume that the SM cross section values can vary by up to $50 \%$ of the our SM cross sections for both processes. We can observe from calculations that changes in the SM cross section do not significantly affect the sensitivity on the anomalous couplings. For example, the SM cross section of the process $\gamma \gamma \rightarrow \tau^{+} \tau^{-}$with $\sqrt{s}=$ $3 \mathrm{TeV}$ is $\sigma_{\mathrm{SM}}=1.97 \mathrm{pb}$. The sensitivity value obtained in

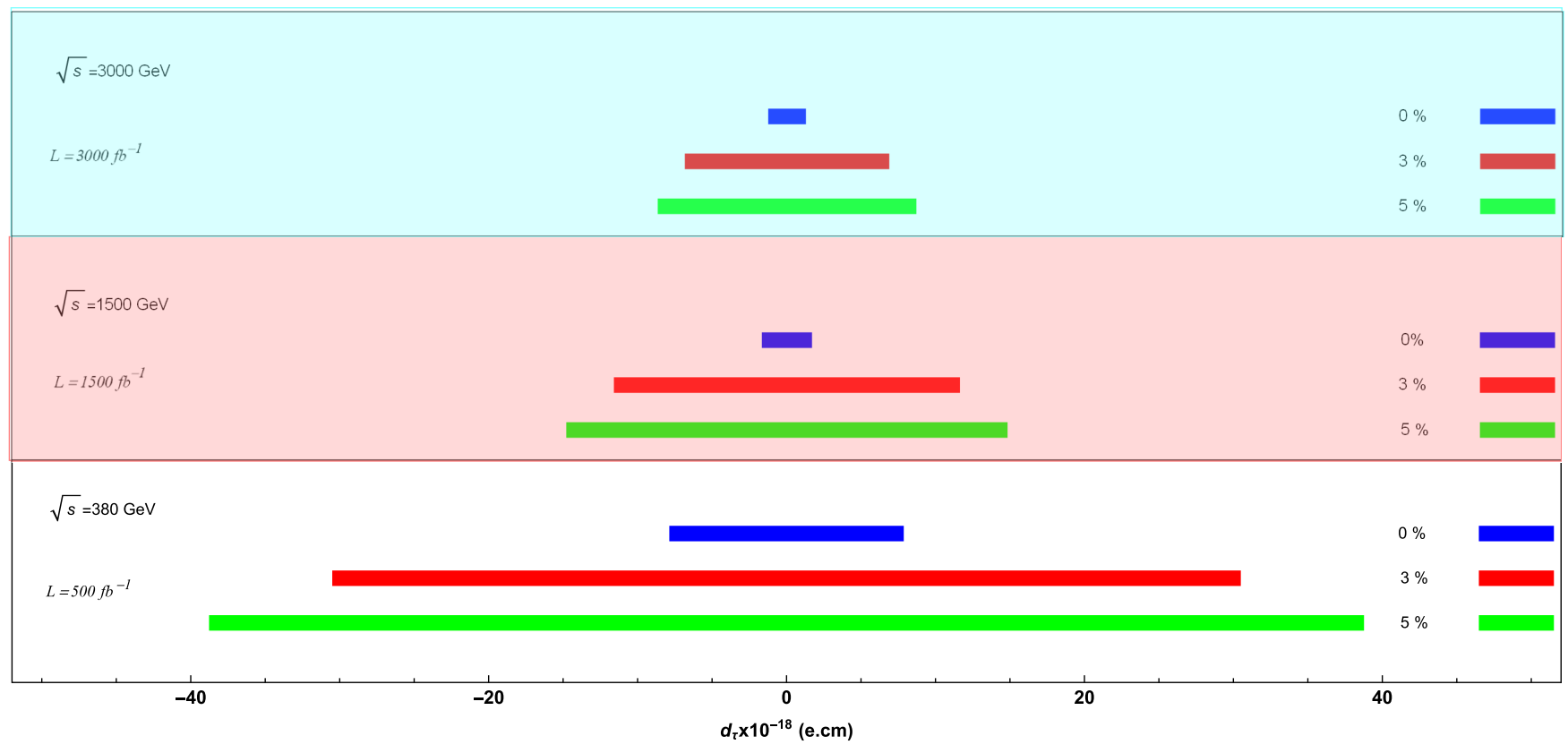

FIG. 14. Same as in Fig. 12, but for $\tilde{d}_{\tau}$. 
TABLE III. Sensitivity on the $\tilde{a}_{\tau}$ magnetic moment and the $\tilde{d}_{\tau}$ electric dipole moment for $\sqrt{s}=380 \mathrm{GeV}$ and $\mathcal{L}=10,50,100$, $300,500 \mathrm{fb}^{-1}$ at $95 \%$ C.L. through the process $\gamma \gamma \rightarrow \tau \bar{\tau}$.

\begin{tabular}{lccc}
\hline \hline \multicolumn{3}{c}{$\sqrt{s}=380 \mathrm{GeV}, 95 \%$ C.L. } \\
\hline $\mathcal{L}\left[\mathrm{fb}^{-1}\right]$ & $\delta_{\text {sys }}$ & $\tilde{a}_{\tau}$ & $\mid \tilde{d}_{\tau}($ ecm $) \mid$ \\
\hline 10 & $0 \%$ & {$[-0.00232 ; 0.00095]$} & $1.071 \times 10^{-17}$ \\
10 & $3 \%$ & {$[-0.00603 ; 0.00464]$} & $2.999 \times 10^{-17}$ \\
10 & $5 \%$ & {$[-0.00756 ; 0.00616]$} & $3.826 \times 10^{-17}$ \\
50 & $0 \%$ & {$[-0.00189 ; 0.00052]$} & $8.813 \times 10^{-18}$ \\
50 & $3 \%$ & {$[-0.00603 ; 0.00464]$} & $2.998 \times 10^{-17}$ \\
50 & $5 \%$ & {$[-0.00756 ; 0.00616]$} & $3.825 \times 10^{-17}$ \\
100 & $0 \%$ & {$[-0.00176 ; 0.00039]$} & $8.298 \times 10^{-18}$ \\
100 & $3 \%$ & {$[-0.00603 ; 0.00464]$} & $2.998 \times 10^{-17}$ \\
100 & $5 \%$ & {$[-0.00756 ; 0.00616]$} & $3.825 \times 10^{-17}$ \\
300 & $0 \%$ & {$[-0.00161 ; 0.00025]$} & $7.737 \times 10^{-18}$ \\
300 & $3 \%$ & {$[-0.00603 ; 0.00464]$} & $2.998 \times 10^{-17}$ \\
300 & $5 \%$ & {$[-0.00756 ; 0.00616]$} & $3.825 \times 10^{-17}$ \\
500 & $0 \%$ & {$[-0.00156 ; 0.00019]$} & $7.556 \times 10^{-18}$ \\
500 & $3 \%$ & {$[-0.00603 ; 0.00464]$} & $2.997 \times 10^{-17}$ \\
500 & $5 \%$ & {$[-0.00756 ; 0.00616]$} & $3.825 \times 10^{-17}$ \\
\hline \hline
\end{tabular}

Table $\mathrm{V}$ for the anomalous magnetic moment $\tilde{a}_{\tau}$ with $\mathcal{L}=$ $3000 \mathrm{fb}^{-1}$ is $\tilde{a}_{\tau}=[-0.00015 ; 0.00017]$. However, when we consider that the SM cross section of this process is $50 \%$ higher, the sensitivity value obtained is $\tilde{a}_{\tau}=[-0.00018$; $0.00019]$. We conclude that the sensitivities are not significantly affected due to the uncertainties of the SM. Other

TABLE IV. Sensitivity on the $\tilde{a}_{\tau}$ magnetic moment and the $\tilde{d}_{\tau}$ electric dipole moment for $\sqrt{s}=1.5 \mathrm{TeV}$ and $\mathcal{L}=100,300$, $500,1000,1500 \mathrm{fb}^{-1}$ at $95 \%$ C.L. through the process $\gamma \gamma \rightarrow \tau \bar{\tau}$.

\begin{tabular}{lccc}
\hline \hline & \multicolumn{3}{c}{$\sqrt{s}=1.5 \mathrm{TeV}, 95 \%$ C.L. } \\
\hline $\mathcal{L}\left[\mathrm{fb}^{-1}\right]$ & $\delta_{\text {sys }}$ & $\tilde{a}_{\tau}$ & $\mid \tilde{d}_{\tau}($ ecm $) \mid$ \\
\hline 100 & $0 \%$ & {$[-0.00061 ; 0.00041]$} & $2.796 \times 10^{-18}$ \\
100 & $3 \%$ & {$[-0.00209 ; 0.00189]$} & $1.109 \times 10^{-17}$ \\
100 & $5 \%$ & {$[-0.00267 ; 0.00247]$} & $1.428 \times 10^{-17}$ \\
300 & $0 \%$ & {$[-0.00049 ; 0.00029]$} & $2.1226 \times 10^{-18}$ \\
300 & $3 \%$ & {$[-0.00209 ; 0.00189]$} & $1.108 \times 10^{-17}$ \\
300 & $5 \%$ & {$[-0.00267 ; 0.00247]$} & $1.428 \times 10^{-17}$ \\
500 & $0 \%$ & {$[-0.00044 ; 0.00025]$} & $1.867 \times 10^{-18}$ \\
500 & $3 \%$ & {$[-0.00209 ; 0.00189]$} & $1.108 \times 10^{-17}$ \\
500 & $5 \%$ & {$[-0.00267 ; 0.00247]$} & $1.428 \times 10^{-17}$ \\
1000 & $0 \%$ & {$[-0.00039 ; 0.00019]$} & $1.567 \times 10^{-18}$ \\
1000 & $3 \%$ & {$[-0.00209 ; 0.00189]$} & $1.108 \times 10^{-17}$ \\
1000 & $5 \%$ & {$[-0.00267 ; 0.00247]$} & $1.427 \times 10^{-17}$ \\
1500 & $0 \%$ & {$[-0.00037 ; 0.00017]$} & $1.415 \times 10^{-18}$ \\
1500 & $3 \%$ & {$[-0.00209 ; 0.00189]$} & $1.108 \times 10^{-17}$ \\
1500 & $5 \%$ & {$[-0.00267 ; 0.00247]$} & $1.427 \times 10^{-17}$ \\
\hline \hline
\end{tabular}

TABLE V. Sensitivity on the $\tilde{a}_{\tau}$ magnetic moment and the $\tilde{d}_{\tau}$ electric dipole moment for $\sqrt{s}=3 \mathrm{TeV}$ and $\mathcal{L}=100,500,1000$, 2000, $3000 \mathrm{fb}^{-1}$ at $95 \%$ C.L. through the process $\gamma \gamma \rightarrow \tau \bar{\tau}$.

\begin{tabular}{lccc}
\hline \hline & \multicolumn{3}{c}{$\sqrt{s}=3 \mathrm{TeV}, 95 \%$ C.L. } \\
\hline $\mathcal{L}\left[\mathrm{fb}^{-1}\right]$ & $\delta_{\text {sys }}$ & $\tilde{a}_{\tau}$ & $\mid \tilde{d}_{\tau}($ ecm $) \mid$ \\
\hline 100 & $0 \%$ & {$[-0.00037 ; 0.00039]$} & $2.116 \times 10^{-18}$ \\
100 & $3 \%$ & {$[-0.00114 ; 0.00115]$} & $6.342 \times 10^{-18}$ \\
100 & $5 \%$ & {$[-0.00147 ; 0.00148]$} & $8.153 \times 10^{-18}$ \\
500 & $0 \%$ & {$[-0.00025 ; 0.00026]$} & $1.415 \times 10^{-18}$ \\
500 & $3 \%$ & {$[-0.00114 ; 0.00115]$} & $6.335 \times 10^{-18}$ \\
500 & $5 \%$ & {$[-0.00147 ; 0.00148]$} & $8.153 \times 10^{-18}$ \\
1000 & $0 \%$ & {$[-0.00020 ; 0.00022]$} & $1.190 \times 10^{-18}$ \\
1000 & $3 \%$ & {$[-0.00114 ; 0.00115]$} & $6.334 \times 10^{-18}$ \\
1000 & $5 \%$ & {$[-0.00147 ; 0.00148]$} & $8.153 \times 10^{-18}$ \\
2000 & $0 \%$ & {$[-0.00017 ; 0.00018]$} & $1.000 \times 10^{-18}$ \\
2000 & $3 \%$ & {$[-0.00114 ; 0.00115]$} & $6.334 \times 10^{-18}$ \\
2000 & $5 \%$ & {$[-0.00147 ; 0.00148]$} & $8.153 \times 10^{-18}$ \\
3000 & $0 \%$ & {$[-0.00015 ; 0.00017]$} & $9.040 \times 10^{-19}$ \\
3000 & $3 \%$ & {$[-0.00114 ; 0.00115]$} & $6.334 \times 10^{-18}$ \\
3000 & $5 \%$ & {$[-0.00147 ; 0.00148]$} & $8.153 \times 10^{-18}$ \\
\hline \hline
\end{tabular}

center-of-mass energies and luminosities produce similar results.

\section{C. $\gamma \gamma \rightarrow \tau^{+} \tau^{-} \gamma$ cross section}

Experimentally, the processes involving the single photon in the final state $\left(\tau^{+} \tau^{-} \gamma\right)$ can potentially be distinguished from background. Furthermore, the anomalous $\tau^{+} \tau^{-} \gamma$ coupling can be analyzed through the process $e^{+} e^{-} \rightarrow \tau^{+} \tau^{-}$at the linear colliders. This process receives contributions from both anomalous $\tau^{+} \tau^{-} \gamma$ and $\tau^{+} \tau^{-} Z$ couplings. However, the processes $\gamma \gamma \rightarrow \tau^{+} \tau^{-}$and $\gamma \gamma \rightarrow \tau^{+} \tau^{-} \gamma$ isolate the $\tau^{+} \tau^{-} \gamma$ coupling, and thus the $\tau^{+} \tau^{-} \gamma$ coupling and the $\tau^{+} \tau^{-} Z$ coupling may be analyzed separately. In general, anomalous values of $\tilde{a}_{\tau}$ and $\tilde{d}_{\tau}$ tend to increase the cross section for the process $\gamma \gamma \rightarrow \tau^{+} \tau^{-} \gamma$, especially for photons with high energy that are well isolated from the decay products of the $\tau$ 's [43]. Additionally, the single photon in the final state has the advantage of being identifiable with high efficiency and purity.

These processes may also provide a clear signal in the detector for new physics and for new phenomena such as the fermion dipole moments. Also, the selection criteria used for the analysis allows searching for events with single-photon characteristics.

We now turn our attention to the process $\gamma \gamma \rightarrow \tau^{+} \tau^{-} \gamma$ at a future $e^{+} e^{-}$collider. For the calculation of the total cross section of $\gamma \gamma \rightarrow \tau^{+} \tau^{-} \gamma$, the analytical expression for the amplitude square is quite lengthy so we do not present it here. Instead, we present numerical fit functions for the total cross sections with respect to center-of-mass energy and in terms of the parameters $\kappa$ and $\tilde{\kappa}$. Furthermore, in the 
case of the process $\gamma \gamma \rightarrow \tau^{+} \tau^{-} \gamma$, we used the following kinematic cuts to reduce the background and to maximize the signal sensitivity:

$$
\begin{aligned}
& p_{t}^{\gamma}> 20 \mathrm{GeV}, \quad\left|\eta^{\gamma}\right|<2.5, \\
& p_{t}^{\tau, \bar{\tau}}>20 \mathrm{GeV}, \quad\left|\eta^{\tau, \bar{\tau}}\right|<2.5, \\
& \Delta R(\tau, \gamma)>0.4, \\
& \Delta R(\tau, \bar{\tau})>0.4, \\
& \Delta R(\bar{\tau}, \gamma)>0.4 .
\end{aligned}
$$

These cuts are applied to the photon transverse momentum $p_{t}^{\gamma}$, to the photon pseudorapidity $\eta^{\gamma}$, which reduces the contamination from other particles misidentified as photons, to the $\tau$ transverse momentum $p_{t}^{\tau, \bar{\tau}}$ for the final state particles, to the $\tau$ pseudorapidity $\eta^{\tau}$ which reduces the contamination from other particles misidentified as $\tau$ and to $\Delta R(\tau, \gamma), \Delta R(\tau, \bar{\tau})$, and $\Delta R(\bar{\tau}, \gamma)$ which give the separation of the final state particles. In conclusion, by using the cuts given in Eq. (24), we have taken into account isolation criteria to optimize the signal to the particles of the $\tau^{+} \tau^{-} \gamma$ final state. The cases considered are the following:

(i) For $\sqrt{s}=380 \mathrm{GeV}$,

$$
\begin{aligned}
\sigma(\kappa, \tilde{\kappa})= & {\left[\left(3.22 \times 10^{7}\right) \kappa^{6}+\left(3.22 \times 10^{7}\right) \tilde{\kappa}^{6}+\left(4.08 \times 10^{4}\right) \kappa^{5}+\left(8.78 \times 10^{7}\right) \kappa^{4} \tilde{\kappa}^{2}+\left(5.56 \times 10^{5}\right) \kappa^{3} \tilde{\kappa}^{2}\right.} \\
& +\left(8.78 \times 10^{7}\right) \kappa^{2} \tilde{\kappa}^{4}+\left(6.94 \times 10^{5}\right) \kappa^{2} \tilde{\kappa}^{2}+\left(2.88 \times 10^{5}\right) \kappa \tilde{\kappa}^{4}+\left(1.52 \times 10^{3}\right) \kappa \tilde{\kappa}^{2}+\left(3.36 \times 10^{5}\right) \kappa^{4} \\
& \left.+\left(3.43 \times 10^{5}\right) \tilde{\kappa}^{4}+\left(1.87 \times 10^{3}\right) \kappa^{3}+\left(1.24 \times 10^{3}\right) \kappa^{2}+\left(1.24 \times 10^{3}\right) \tilde{\kappa}^{2}+(0.515) \kappa+0.21\right](\mathrm{pb}) .
\end{aligned}
$$

(ii) For $\sqrt{s}=1500 \mathrm{GeV}$,

$$
\begin{aligned}
\sigma(\kappa, \tilde{\kappa})= & {\left[\left(8.29 \times 10^{9}\right) \kappa^{6}+\left(8.29 \times 10^{9}\right) \tilde{\kappa}^{6}+\left(8.65 \times 10^{6}\right) \kappa^{5}+\left(2.43 \times 10^{10}\right) \kappa^{4} \tilde{\kappa}^{2}+\left(3.26 \times 10^{7}\right) \kappa^{3} \tilde{\kappa}^{2}\right.} \\
& +\left(2.41 \times 10^{10}\right) \kappa^{2} \tilde{\kappa}^{4}+\left(2.43 \times 10^{7}\right) \kappa^{2} \tilde{\kappa}^{2}+\left(2.20 \times 10^{7}\right) \kappa \tilde{\kappa}^{4}+\left(1.52 \times 10^{3}\right) \kappa \tilde{\kappa}^{2}+\left(4.63 \times 10^{7}\right) \kappa^{5} \\
& +\left(1.15 \times 10^{7}\right) \tilde{\kappa}^{4}+\left(1.15 \times 10^{7}\right) \kappa^{4}+\left(3.90 \times 10^{3}\right) \kappa^{3}+\left(3.74 \times 10^{3}\right) \kappa^{2}+\left(3.74 \times 10^{3}\right) \tilde{\kappa}^{2} \\
& +(0.31) \kappa+0.116](\mathrm{pb}) .
\end{aligned}
$$

(iii) For $\sqrt{s}=3000 \mathrm{GeV}$,

$$
\begin{aligned}
\sigma(\kappa, \tilde{\kappa})= & {\left[\left(1.33 \times 10^{11}\right) \kappa^{6}+\left(1.33 \times 10^{11}\right) \tilde{\kappa}^{6}+\left(4.04 \times 10^{11}\right) \kappa^{4} \tilde{\kappa}^{2}+\left(3.32 \times 10^{7}\right) \kappa^{3} \tilde{\kappa}^{2}+\left(4.04 \times 10^{11}\right) \kappa^{2} \tilde{\kappa}^{4}\right.} \\
& +\left(1.09 \times 10^{8}\right) \kappa^{2} \tilde{\kappa}^{2}+\left(3.55 \times 10^{7}\right) \kappa \tilde{\kappa}^{4}+\left(1.03 \times 10^{4}\right) \kappa \tilde{\kappa}^{2}+\left(4.63 \times 10^{7}\right) \kappa^{5}+\left(5.58 \times 10^{7}\right) \tilde{\kappa}^{4} \\
& \left.+\left(5.58 \times 10^{7}\right) \kappa^{4}+\left(1.46 \times 10^{3}\right) \kappa^{3}+\left(4.92 \times 10^{3}\right) \kappa^{2}+\left(4.92 \times 10^{3}\right) \tilde{\kappa}^{2}+(0.47) \kappa+0.052\right](\mathrm{pb}) .
\end{aligned}
$$

It is worth mentioning that in the equations for the total cross section, (25)-(27), the terms with $\kappa(\tilde{\kappa})$ give the anomalous contribution, while the independent terms of $\kappa(\tilde{\kappa})$ correspond to the cross section at $\kappa=\tilde{\kappa}=0$ and represent the SM cross-section magnitude.

\section{Sensitivity on $\tilde{\boldsymbol{a}}_{\tau}$ and $\tilde{\boldsymbol{d}}_{\tau}$ through $\gamma \gamma \rightarrow \tau^{+} \tau^{-} \gamma$ at the CLIC}

We now focus our attention on the numerical calculation for the total cross section and for the electromagnetic dipole moments of the $\tau$-lepton. We start with Eqs. (25)-(27), adopting the collider parameters of $\sqrt{s}=380,1500$, $3000 \mathrm{GeV}$ for the center-of-mass energy and $\mathcal{L}=10$, $50,100,300,500,1000,1500,2000,3000 \mathrm{fb}^{-1}$ for the integrated luminosity of data. We then apply kinematic cuts given by Eq. (24) to optimize the signal and to reduce the background. An important part of our study is the inclusion of systematic uncertainties of $\delta_{\text {sys }}=0 \%, 3 \%, 5 \%$.

Figures 15 and 16 present the results for the total cross section for the $\gamma \gamma \rightarrow \tau^{+} \tau^{-} \gamma$ scenario, where the total cross sections for $\sigma\left(\gamma \gamma \rightarrow \tau^{+} \tau^{-} \gamma\right)$ vs $\kappa(\tilde{\kappa})$ are shown for $\sqrt{s}=380,1500,3000 \mathrm{GeV}$, respectively. In both cases, the total cross section $\sigma(\sqrt{s}, \kappa, \tilde{\kappa})$ presents a clear dependence with respect to $\sqrt{s}$, as well as with the anomalous parameters $\kappa$ and $\tilde{\kappa}$. The total cross section for $\gamma \gamma \rightarrow \tau^{+} \tau^{-}$is 10 times greater than $\gamma \gamma \rightarrow \tau^{+} \tau^{-} \gamma$. The effects of $\kappa$ and $\tilde{\kappa}$ on the total cross section $\sigma_{\gamma \gamma \rightarrow \tau^{+} \tau^{-} \gamma}(\sqrt{s}, \kappa, \tilde{\kappa})$ are shown in Figs. 17-19. 


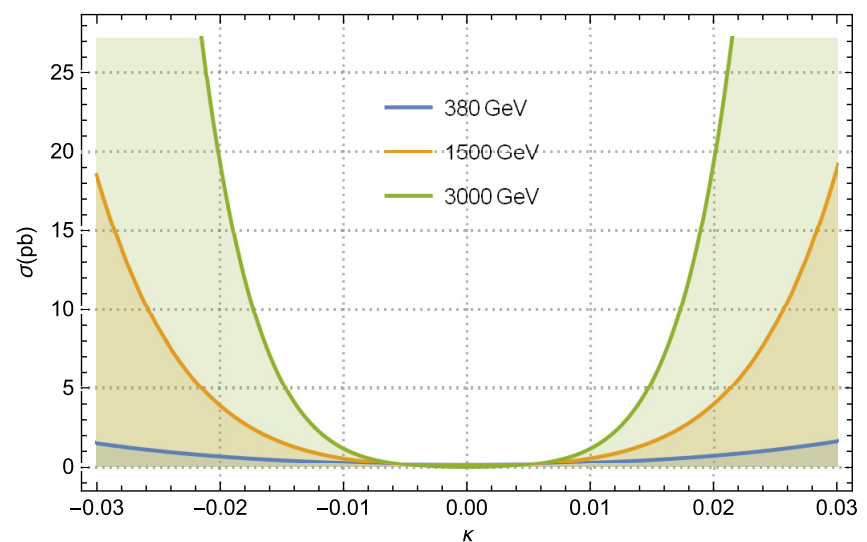

FIG. 15. The total cross sections of the process $\gamma \gamma \rightarrow \tau^{+} \tau^{-} \gamma$ as a function of $\kappa$ for center-of-mass energies of $\sqrt{s}=380,1500$, $3000 \mathrm{GeV}$.

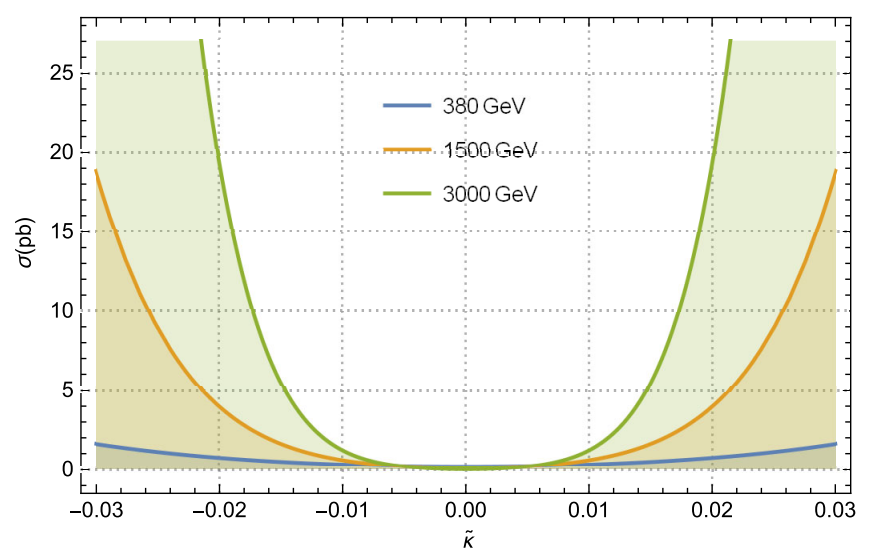

FIG. 16. Same as in Fig. 15, but for $\tilde{\kappa}$.

As shown in Figs. 20-22, the contour plots on $\tilde{\kappa}$ and $\tilde{\kappa}$ at the $95 \%$ C.L. are obtained using Eqs. (25)-(27). Integrate luminosities are $\mathcal{L}=10,100,500,1500,3000 \mathrm{fb}^{-1}$ with $\sqrt{s}=380,1500,3000 \mathrm{GeV}$, respectively. Sensitivity improvement for $\tilde{\kappa}$ and $\tilde{\kappa}$ is obtained using high energy and high luminosity.

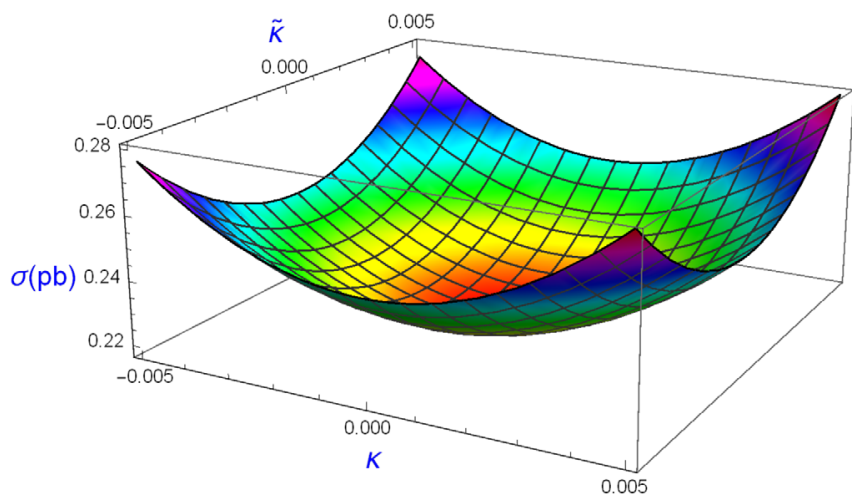

FIG. 17. The total cross sections of the process $\gamma \gamma \rightarrow \tau^{+} \tau^{-} \gamma$ as a function of $\kappa$ and $\tilde{\kappa}$ for center-of-mass energy of $\sqrt{s}=380 \mathrm{GeV}$.

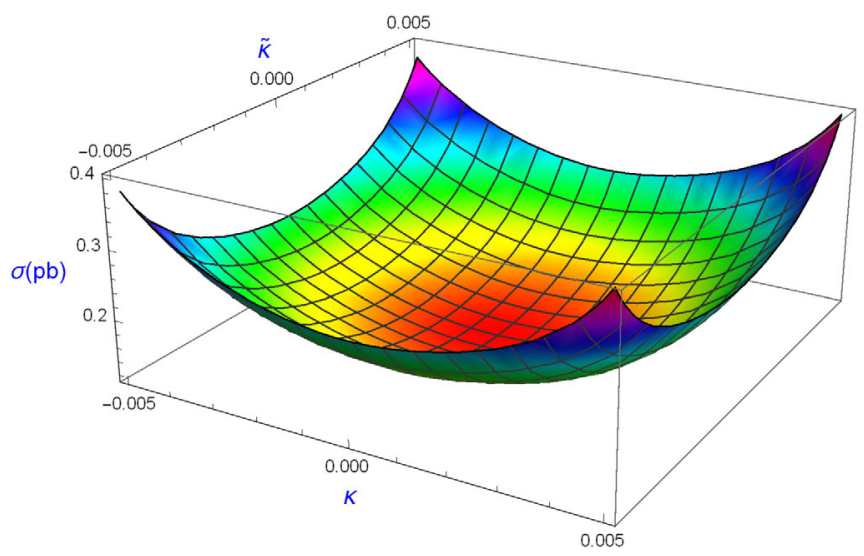

FIG. 18. Same as in Fig. 17, but for $\sqrt{s}=1500 \mathrm{GeV}$.

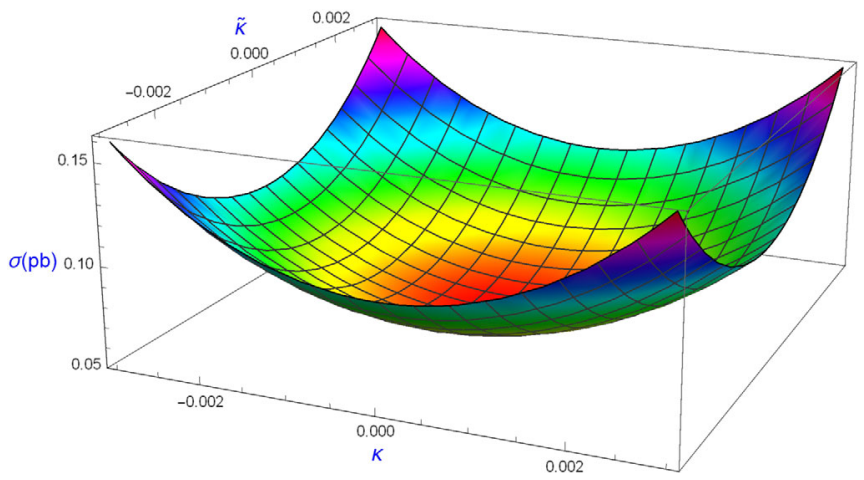

FIG. 19. Same as in Fig. 17, but for $\sqrt{s}=3000 \mathrm{GeV}$.

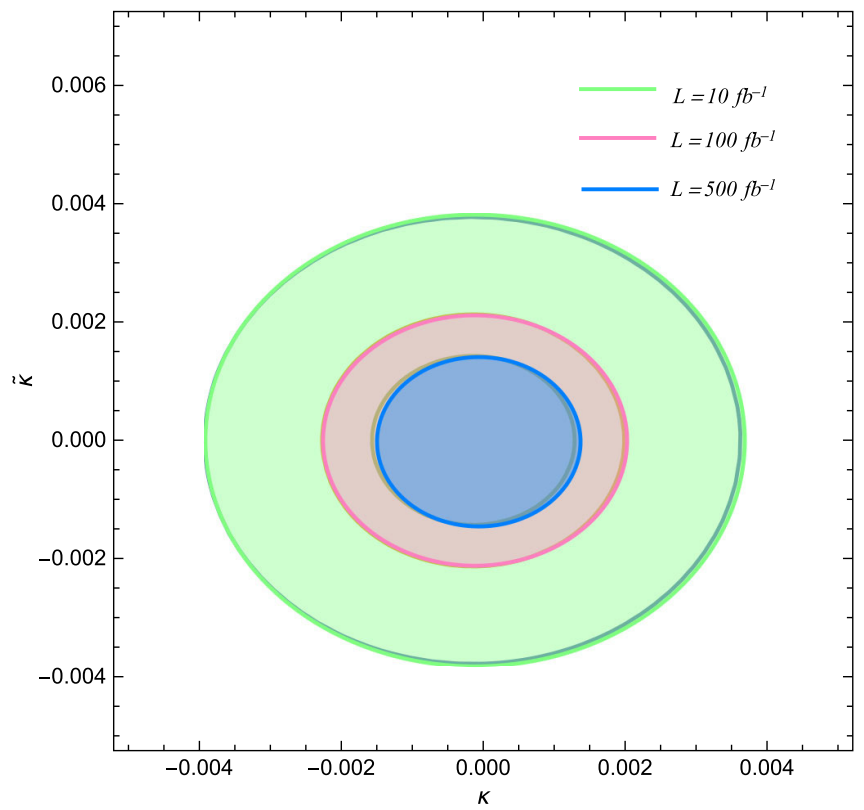

FIG. 20. Sensitivity contours at the $95 \%$ C.L. in the $(\kappa-\tilde{\kappa})$ plane for the process $\gamma \gamma \rightarrow \tau^{+} \tau^{-} \gamma$ with the $\mathcal{L}=10,100,500 \mathrm{fb}^{-1}$ and for center-of-mass energy of $\sqrt{s}=380 \mathrm{GeV}$. 


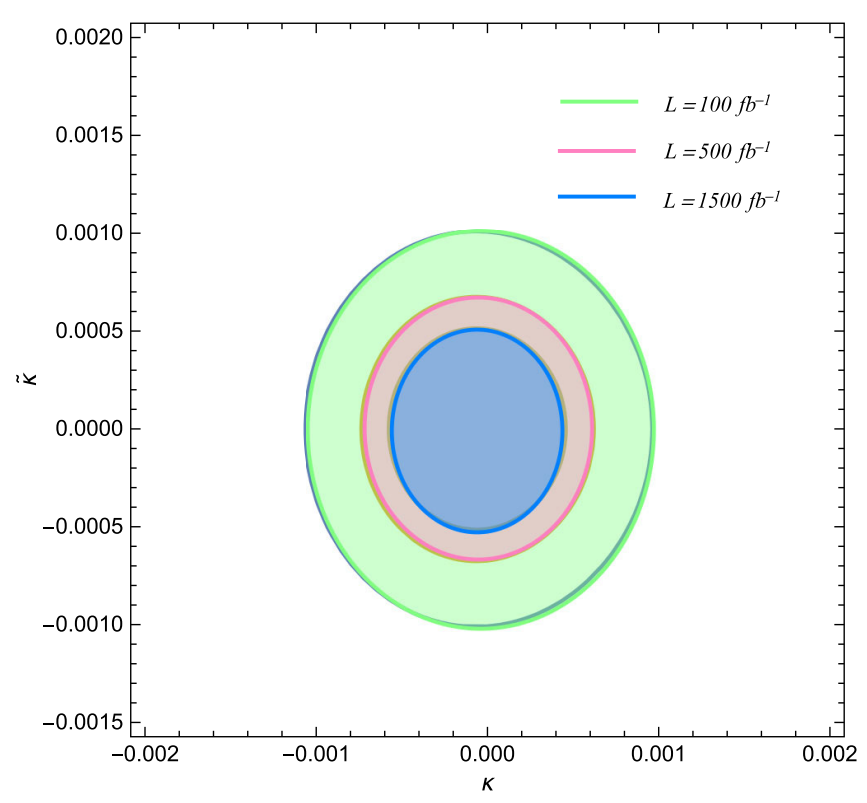

FIG. 21. Same as in Fig. 20, but for $\mathcal{L}=100,500,1500 \mathrm{fb}^{-1}$ and for center-of-mass energy of $\sqrt{s}=1500 \mathrm{GeV}$.

The estimated sensitivity of the CLIC for $\tilde{a}_{\tau}\left(\tilde{d}_{\tau}\right)$ at 95\% C.L., as well as for different center-of-mass energies, luminosities, and systematic errors, is illustrated in Figs. 23-26. The sensitivity is $1-2$ orders of magnitude better for all couplings than that expected at the current colliders (see Table I), and the potential to thoroughly analyze $\tilde{a}_{\tau}$ and $\tilde{d}_{\tau}$ improves at larger $\sqrt{s}$ and $\mathcal{L}$.

To summarize our set of results, Tables VI-VIII show the sensitivity corresponding to the magnetic and electric dipole moments of the $\tau$-lepton, via the $\gamma \gamma \rightarrow \tau^{+} \tau^{-} \gamma$ mode.

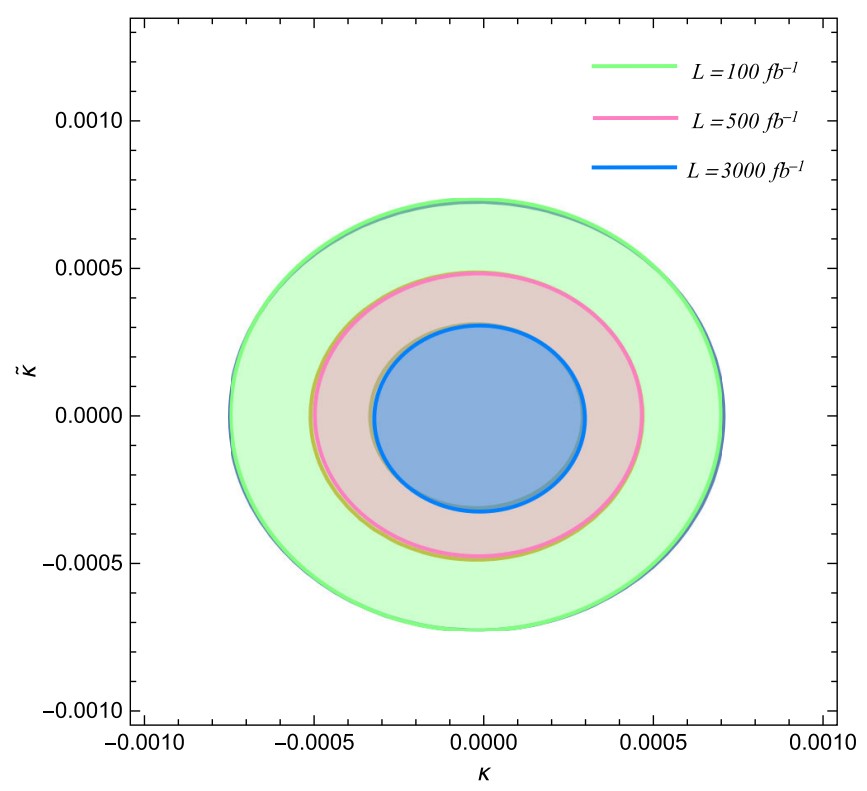

FIG. 22. Same as in Fig. 20, but for $\mathcal{L}=100,500,3000 \mathrm{fb}^{-1}$ and for center-of-mass energy of $\sqrt{s}=3000 \mathrm{GeV}$.

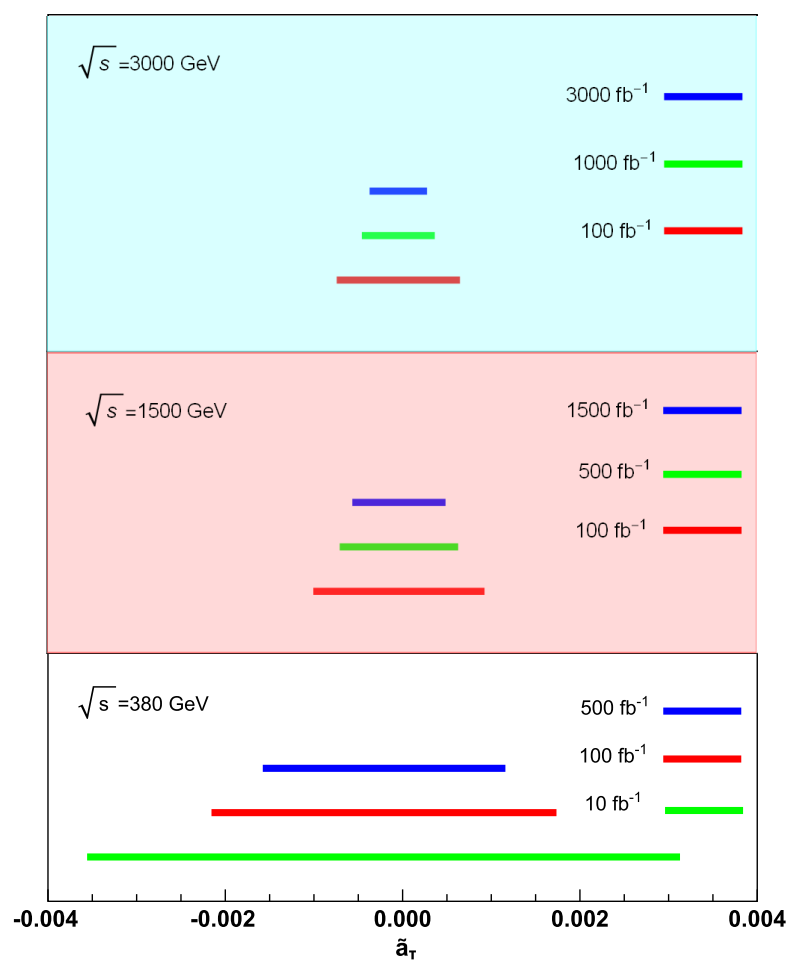

FIG. 23. Comparison of precisions on $\tilde{a}_{\tau}$ in the process $\gamma \gamma \rightarrow$ $\tau^{+} \tau^{-} \gamma$ expected at the CLIC. We assume luminosities of the following: Top panel: $\mathcal{L}=100,1000,3000 \mathrm{fb}^{-1}$ and $\sqrt{s}=$ $3000 \mathrm{GeV}$. Central panel: $\mathcal{L}=100,500,1500 \mathrm{fb}^{-1}$ and $\sqrt{s}=$ $1500 \mathrm{GeV}$. Bottom panel: $\mathcal{L}=10, \quad 100,500 \mathrm{fb}^{-1}$ and $\sqrt{s}=380 \mathrm{GeV}$.

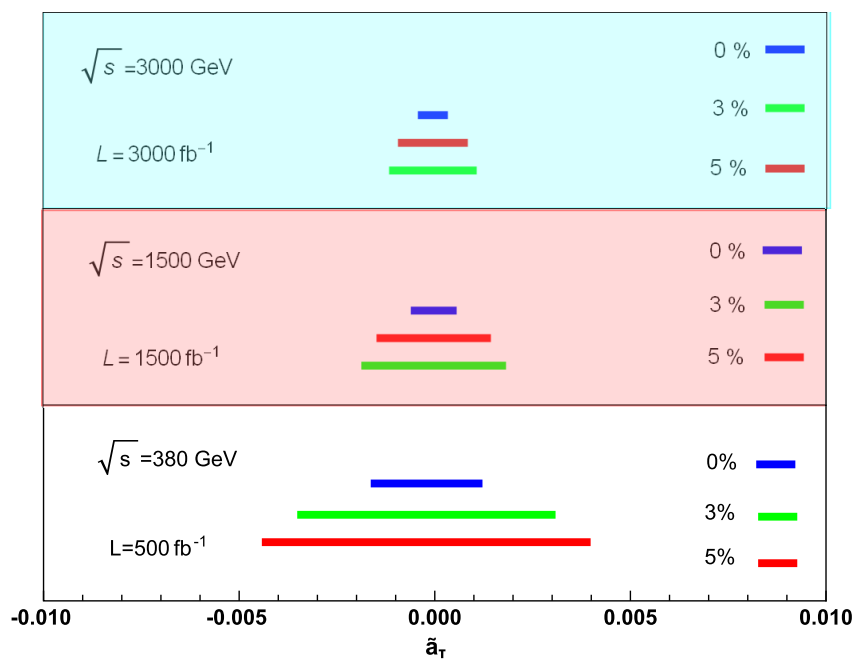

FIG. 24. Same as in Fig. 23, but for the following: Top panel: $\delta_{\text {sys }}=0 \%, 3 \%, 5 \%$ with $\mathcal{L}=3000 \mathrm{fb}^{-1}$ and $\sqrt{s}=3000 \mathrm{GeV}$. Central panel: $\delta_{\text {sys }}=0 \%, 3 \%, 5 \%$ with $\mathcal{L}=1500 \mathrm{fb}^{-1}$ and $\sqrt{s}=1500 \mathrm{GeV}$. Bottom panel: $\delta_{\text {sys }}=0 \%, 3 \%, 5 \%$ with $\mathcal{L}=$ $500 \mathrm{fb}^{-1}$ and $\sqrt{s}=380 \mathrm{GeV}$. 


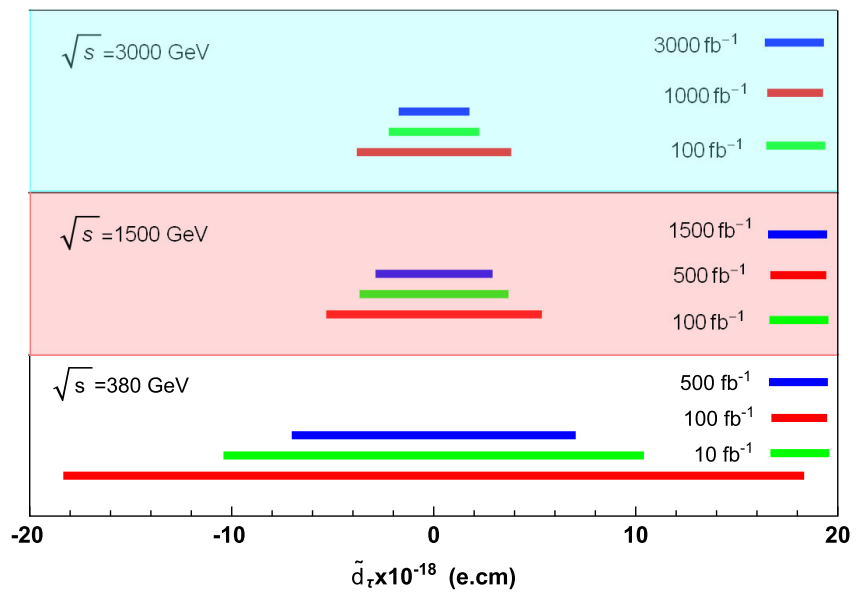

FIG. 25. Same as in Fig. 23, but for $\tilde{d}_{\tau}$.

The assumed center-of-mass energies and luminosities are $\sqrt{s}=380,1500,3000$ and $\mathcal{L}=10,50,100,300,500$, $1000,1500,2000,3000 \mathrm{fb}^{-1}$, respectively, and only one anomalous $\tilde{a}_{\tau}\left(\tilde{d}_{\tau}\right)$ coupling was varied at a time to estimate its value. In this study, the sensitivities are based on the systematic errors of $\delta_{\text {sys }}=0 \%, 3 \%, 5 \%$, and our best sensitivity estimates are $-0.00033 \leq \tilde{a}_{\tau} \leq 0.00023$ and $\left|\tilde{d}_{\tau}(\mathrm{ecm})\right|=1.546 \times 10^{-18}$, respectively. These sensitivity estimates are 1 order of magnitude weaker than those corresponding to the $\gamma \gamma \rightarrow \tau^{+} \tau^{-}$mode (see Sec. II B, Tables III-V), but stronger than those reported by BELLE, DELPHI, L3, and OPAL Collaborations (see Table I).
TABLE VI. Sensitivity on the $\tilde{a}_{\tau}$ magnetic moment and the $\tilde{d}_{\tau}$ electric dipole moment for $\sqrt{s}=380 \mathrm{GeV}$ and $\mathcal{L}=10,50,100$, $300,500 \mathrm{fb}^{-1}$ at $95 \%$ C.L. through the process $\gamma \gamma \rightarrow \tau \bar{\tau} \gamma$.

\begin{tabular}{lccc}
\hline \hline & \multicolumn{3}{c}{$=380 \mathrm{GeV}, 95 \%$ C.L. } \\
\hline $\mathcal{L}\left[\mathrm{fb}^{-1}\right]$ & $\delta_{\text {sys }}$ & $\tilde{a}_{\tau}$ & $\mid \tilde{d}_{\tau}($ ecm $) \mid$ \\
\hline 10 & $0 \%$ & {$[-0.00351 ; 0.00308]$} & $1.813 \times 10^{-17}$ \\
10 & $3 \%$ & {$[-0.00375 ; 0.00332]$} & $1.977 \times 10^{-17}$ \\
10 & $5 \%$ & {$[-0.00449 ; 0.00405]$} & $2.385 \times 10^{-17}$ \\
50 & $0 \%$ & {$[-0.00245 ; 0.00203]$} & $1.213 \times 10^{-17}$ \\
50 & $3 \%$ & {$[-0.00348 ; 0.00305]$} & $1.829 \times 10^{-17}$ \\
50 & $5 \%$ & {$[-0.00435 ; 0.00391]$} & $2.306 \times 10^{-17}$ \\
100 & $0 \%$ & {$[-0.00211 ; 0.00169]$} & $1.020 \times 10^{-17}$ \\
100 & $3 \%$ & {$[-0.00344 ; 0.00301]$} & $1.807 \times 10^{-17}$ \\
100 & $5 \%$ & {$[-0.00433 ; 0.00389]$} & $2.296 \times 10^{-17}$ \\
300 & $0 \%$ & {$[-0.00169 ; 0.00127]$} & $7.758 \times 10^{-18}$ \\
300 & $3 \%$ & {$[-0.00342 ; 0.00299]$} & $1.792 \times 10^{-17}$ \\
300 & $5 \%$ & {$[-0.00432 ; 0.00388]$} & $2.289 \times 10^{-17}$ \\
500 & $0 \%$ & {$[-0.00153 ; 0.00111]$} & $6.828 \times 10^{-18}$ \\
500 & $3 \%$ & {$[-0.00341 ; 0.00298]$} & $1.789 \times 10^{-17}$ \\
500 & $5 \%$ & {$[-0.00432 ; 0.00388]$} & $2.288 \times 10^{-17}$ \\
\hline \hline
\end{tabular}

Finally, we determine how realistic our estimated values are for the error parameter delta for the process $\gamma \gamma \rightarrow \tau^{-} \tau^{+} \gamma$. Discussions similar to the process $\gamma \gamma \rightarrow$ $\tau^{-} \tau^{+}$are also valid for the aforementioned process. We understand that changes in the SM cross section do not significantly affect the sensitivity estimates on the anomalous parameters.

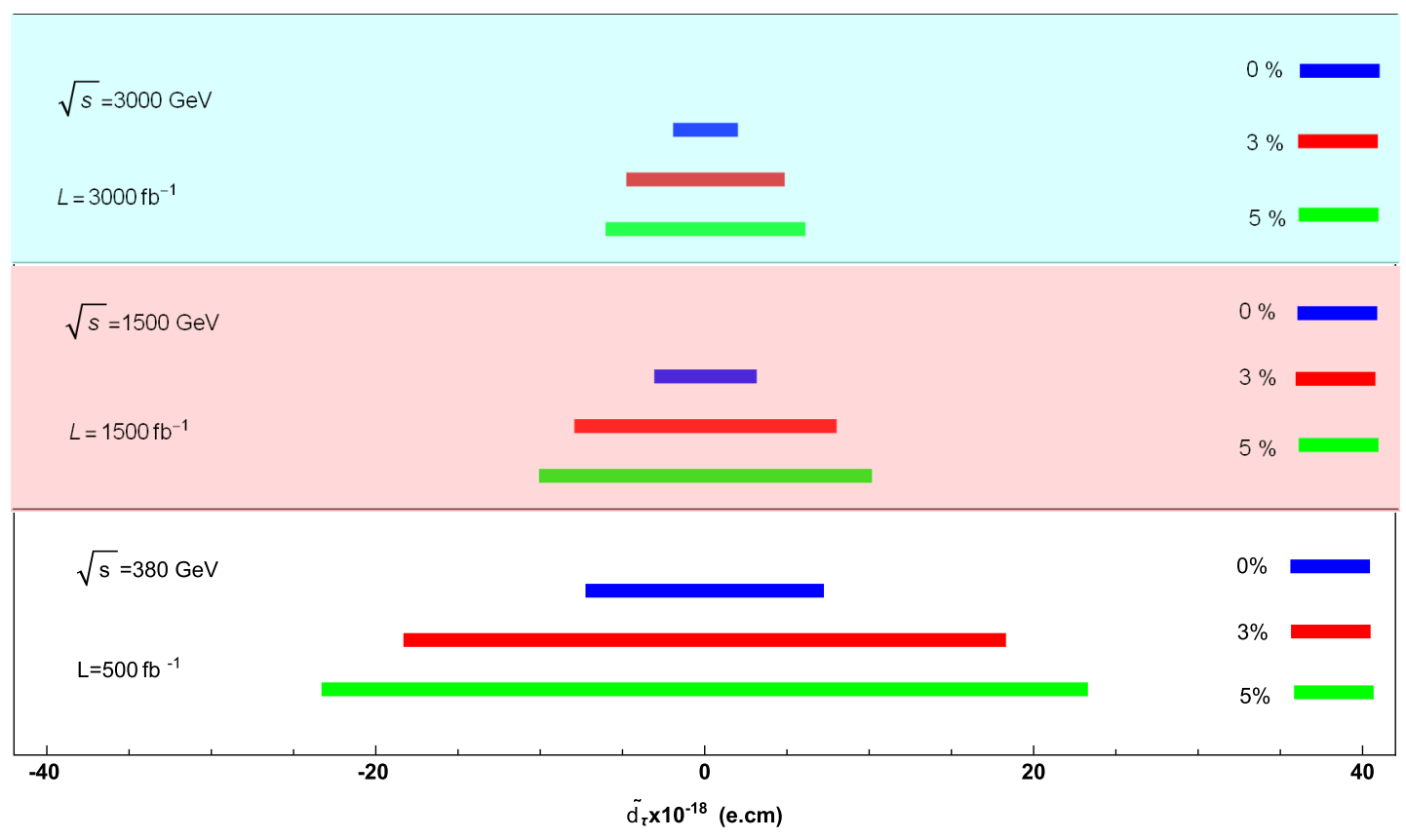

FIG. 26. Same as in Fig. 24, but for $\tilde{d}_{\tau}$. 
TABLE VII. Sensitivity on the $\tilde{a}_{\tau}$ magnetic moment and the $\tilde{d}_{\tau}$ electric dipole moment for $\sqrt{s}=1.5 \mathrm{TeV}$ and $\mathcal{L}=100,300$, $500,1000,1500 \mathrm{fb}^{-1}$ at $95 \%$ C.L. through the process $\gamma \gamma \rightarrow \tau \bar{\tau} \gamma$.

\begin{tabular}{lccc}
\hline \hline & \multicolumn{3}{c}{$\sqrt{s}=1.5 \mathrm{TeV}, 95 \%$ C.L. } \\
\hline $\mathcal{L}\left[\mathrm{fb}^{-1}\right]$ & $\delta_{\text {sys }}$ & $\tilde{a}_{\tau}$ & $\mid \tilde{d}_{\tau}($ ecm $) \mid$ \\
\hline 100 & $0 \%$ & {$[-0.00096 ; 0.00088]$} & $5.121 \times 10^{-18}$ \\
100 & $3 \%$ & {$[-0.00142 ; 0.00134]$} & $7.695 \times 10^{-18}$ \\
100 & $5 \%$ & {$[-0.00179 ; 0.00171]$} & $9.756 \times 10^{-18}$ \\
300 & $0 \%$ & {$[-0.00074 ; 0.00066]$} & $3.926 \times 10^{-18}$ \\
300 & $3 \%$ & {$[-0.00140 ; 0.00132]$} & $7.582 \times 10^{-18}$ \\
300 & $5 \%$ & {$[-0.00178 ; 0.00170]$} & $9.702 \times 10^{-18}$ \\
500 & $0 \%$ & {$[-0.00066 ; 0.00058]$} & $3.475 \times 10^{-18}$ \\
500 & $3 \%$ & {$[-0.00140 ; 0.00131]$} & $7.559 \times 10^{-18}$ \\
500 & $5 \%$ & {$[-0.00178 ; 0.00170]$} & $9.691 \times 10^{-18}$ \\
1000 & $0 \%$ & {$[-0.00057 ; 0.00049]$} & $2.952 \times 10^{-18}$ \\
1000 & $3 \%$ & {$[-0.00139 ; 0.00131]$} & $7.541 \times 10^{-18}$ \\
1000 & $5 \%$ & {$[-0.00178 ; 0.00170]$} & $9.683 \times 10^{-18}$ \\
1500 & $0 \%$ & {$[-0.00052 ; 0.00044]$} & $2.688 \times 10^{-18}$ \\
1500 & $3 \%$ & {$[-0.00139 ; 0.00131]$} & $7.535 \times 10^{-18}$ \\
1500 & $5 \%$ & {$[-0.00178 ; 0.00170]$} & $9.680 \times 10^{-18}$ \\
\hline \hline
\end{tabular}

\section{CONCLUSIONS}

We have presented a complete study of the total cross section as well as of the anomalous magnetic and electric dipole moments of the $\tau$-lepton for $\gamma \gamma \rightarrow \tau^{+} \tau^{-}$and $\gamma \gamma \rightarrow$ $\tau^{+} \tau^{-} \gamma$ at the CLIC. We consider the parameters $\tilde{a}_{\tau}$ and $\tilde{d}_{\tau}$ for both processes, as well as collider parameters $\sqrt{s}$ and $\mathcal{L}$. Furthermore, we apply a set of appropriate cuts given by Eqs. (12) and (24) to reduce the background and optimize the signal sensitivity to the particles of the $\tau^{+} \tau^{-}\left(\tau^{+} \tau^{-} \gamma\right)$ final state.

In general terms, our study shows that the process $\gamma \gamma \rightarrow$ $\tau^{+} \tau^{-}$with two- $\tau$ in the final state is more representative and shows better sensitivity in both the total cross section and in the electromagnetic dipole moments when compared to the process $\gamma \gamma \rightarrow \tau^{+} \tau^{-} \gamma$ in the entire range of center-of-mass energies and luminosities of the future CLIC. Another important factor for distinguishing the sensitivity in our results is the incorporation of the systematic uncertainties of $\delta_{\text {sys }}=0 \%, 3 \%, 5 \%$.

We have also shown that the two-photon $\gamma \gamma \rightarrow \tau^{+} \tau^{-}$and $\gamma \gamma \rightarrow \tau^{+} \tau^{-} \gamma$ processes at the CLIC lead to an improvement in the existing sensitivity estimates on the $\tilde{a}_{\tau}$ and $\tilde{d}_{\tau}$. We present an optimistic scenario regarding the potential
TABLE VIII. Sensitivity on the $\tilde{a}_{\tau}$ magnetic moment and the $\tilde{d}_{\tau}$ electric dipole moment for $\sqrt{s}=3 \mathrm{TeV}$ and $\mathcal{L}=100,500,1000$, 20000, $3000 \mathrm{fb}^{-1}$ at 95\% C.L. through the process $\gamma \gamma \rightarrow \tau \bar{\tau} \gamma$.

\begin{tabular}{lccc}
\hline \hline & \multicolumn{3}{c}{$\sqrt{s}=3 \mathrm{TeV}, 95 \%$ C.L. } \\
\hline $\mathcal{L}\left[\mathrm{fb}^{-1}\right]$ & $\delta_{\text {sys }}$ & $\tilde{a}_{\tau}$ & $\mid \tilde{d}_{\tau}($ ecm $) \mid$ \\
\hline 100 & $0 \%$ & {$[-0.00070 ; 0.00060]$} & $3.612 \times 10^{-18}$ \\
100 & $3 \%$ & {$[-0.00087 ; 0.00078]$} & $4.591 \times 10^{-18}$ \\
100 & $5 \%$ & {$[-0.00108 ; 0.00098]$} & $5.740 \times 10^{-18}$ \\
500 & $0 \%$ & {$[-0.00048 ; 0.00039]$} & $2.418 \times 10^{-18}$ \\
500 & $3 \%$ & {$[-0.00084 ; 0.00075]$} & $4.422 \times 10^{-18}$ \\
500 & $5 \%$ & {$[-0.00106 ; 0.00097]$} & $5.658 \times 10^{-18}$ \\
1000 & $0 \%$ & {$[-0.00041 ; 0.00032]$} & $2.034 \times 10^{-18}$ \\
1000 & $3 \%$ & {$[-0.00084 ; 0.00074]$} & $4.399 \times 10^{-18}$ \\
1000 & $5 \%$ & {$[-0.00106 ; 0.00097]$} & $5.647 \times 10^{-18}$ \\
2000 & $0 \%$ & {$[-0.00035 ; 0.00026]$} & $1.711 \times 10^{-18}$ \\
2000 & $3 \%$ & {$[-0.00083 ; 0.00074]$} & $4.388 \times 10^{-18}$ \\
2000 & $5 \%$ & {$[-0.00106 ; 0.00097]$} & $5.642 \times 10^{-18}$ \\
3000 & $0 \%$ & {$[-0.00033 ; 0.00023]$} & $1.546 \times 10^{-18}$ \\
3000 & $3 \%$ & {$[-0.00083 ; 0.00074]$} & $4.384 \times 10^{-18}$ \\
3000 & $5 \%$ & {$[-0.00106 ; 0.00097]$} & $5.640 \times 10^{-18}$ \\
\hline
\end{tabular}

precision, energy, and luminosity that may be achievable at the future $e^{+} e^{-}$colliders. For the process $\gamma \gamma \rightarrow \tau^{+} \tau^{-}$, we obtain $3.466 \times 10^{2}$ for the upper sensitivity and $0.764 \times 10^{2}$ for the lower sensitivity, showing an improvement when compared to the results published by the DELPHI and BELLE Collaborations for the reaction $e^{+} e^{-} \rightarrow \tau^{+} \tau^{-}[42,45]$. The process $\gamma \gamma \rightarrow \tau^{+} \tau^{-} \gamma$ shows an improvement of the order of $2.060 \times 10^{2}$ and $2.826 \times$ $10^{2}$ for the upper and lower sensitivities, respectively, when compared to the results reported by the L3 and OPAL Collaborations for the process $e^{+} e^{-} \rightarrow \tau^{+} \tau^{-} \gamma$ $[43,44]$, as shown in Table I. Our results indicate that the processes $\gamma \gamma \rightarrow \tau^{+} \tau^{-}$and $\gamma \gamma \rightarrow \tau^{+} \tau^{-} \gamma$ are more suitable for probing the electromagnetic dipole moments of the $\tau$-lepton in future $e^{+} e^{-}$linear colliders such as the CLIC at the $\gamma \gamma$ mode.

\section{ACKNOWLEDGMENTS}

A. G. R. and M. A.H. R acknowledges support from Sistena Nacional de Investigadores (SNI) and Programa de Fortalecimiento de la Calidad en Instituciones Educativas (PROFOCIE, México). 
[1] S. L. Glashow, Nucl. Phys. 22, 579 (1961).

[2] S. Weinberg, Phys. Rev. Lett. 19, 1264 (1967).

[3] A. Salam, in Elementary Particle Theory, edited by N. Svartholm (Almquist and Wiskell, Stockholm, 1968), p. 367.

[4] D. Hanneke, S. Fogwell, and G. Gabrielse, Phys. Rev. Lett. 100, 120801 (2008).

[5] G. Bennett et al., Phys. Rev. D 73, 072003 (2006).

[6] C. Patrignani et al. (Particle Data Group), Chin. Phys. C 40, 100001 (2016).

[7] S. Eidelman and M. Passera, Mod. Phys. Lett. A 22, 159 (2007).

[8] M. A. Samuel, G. Li, and R. Mendel, Phys. Rev. Lett. 67, 668 (1991); 69, 995(E) (1992).

[9] F. Hamzeh and N. F. Nasrallah, Phys. Lett. B 373, 211 (1996).

[10] S. M. Barr and W. Marciano, in CP Violation, edited by C. Jarlskog (World Scientific, Singapore, 1990).

[11] J. H. Christenson, J. W. Cronin, V. L. Fitch, and R. Turlay, Phys. Rev. Lett. 13, 138 (1964).

[12] K. Abe et al., Phys. Rev. Lett. 87, 091802 (2001).

[13] R. Aaij et al. (LHCb Collaboration), J. High Energy Phys. 07 (2014) 041.

[14] W. Bernreuther, A. Brandenburg, and P. Overmann, Phys. Lett. B 391, 413 (1997); 412, 425(E) (1997).

[15] E. O. Iltan, Eur. Phys. J. C 44, 411 (2005).

[16] B. Dutta and R. N. Mohapatra, Phys. Rev. D 68, 113008 (2003).

[17] E. Iltan, Phys. Rev. D 64, 013013 (2001).

[18] E. Iltan, J. High Energy Phys. 05 (2003) 065.

[19] E. Iltan, J. High Energy Phys. 04 (2004) 018.

[20] A. Gutiérrez-Rodríguez, M. A. Hernández-Ruíz, and L. N. Luis-Noriega, Mod. Phys. Lett. A 19, 2227 (2004).

[21] A. Gutiérrez-Rodríguez, M. A. Hernández-Ruíz, and M. A. Pérez, Int. J. Mod. Phys. A 22, 3493 (2007).

[22] A. Gutiérrez-Rodríguez, Mod. Phys. Lett. A 25, 703 (2010).

[23] A. Gutiérrez-Rodríguez, M. A. Hernández-Ruíz, and C. P. Castañeda-Almanza, J. Phys. G 40, 035001 (2013).

[24] M. Köksal, S. C. Inan, A. A. Billur, M. K. Bahar, and Y. Özgüven, arXiv:1711.02405.

[25] Y. Özgüven, S. C. Inan, A. A. Billur, M. Köksal, and M. K. Bahar, Nucl. Phys. B923, 475 (2017).

[26] A. A. Billur and M. Köksal, Phys. Rev. D 89, 037301 (2014).

[27] L. Tabares and O. A. Sampayo, Phys. Rev. D 65, 053012 (2002).

[28] S. Eidelman, D. Epifanov, M. Fael, L. Mercolli, and M. Passera, J. High Energy Phys. 03 (2016) 140.

[29] I. Galon, A. Rajaraman, and T. M. P. Tait, J. High Energy Phys. 12 (2016) 111.

[30] M. A. Arroyo-Ureña, G. Hernández-Tomé, and G. TavaresVelasco, Eur. Phys. J. C 77, 227 (2017).

[31] M. A. Arroyo-Ureña, E. Díaz, O. Meza-Aldama, and G. Tavares-Velasco, Int. J. Mod. Phys. A 32, 1750195 (2017).

[32] X. Chen et al., arXiv:1803.00501.

[33] A. Pich, Prog. Part. Nucl. Phys. 75, 41 (2014).

[34] S. Atag and E. Gurkanli, J. High Energy Phys. 06 (2016) 118.

[35] L. Taylor, Nucl. Phys. B, Proc. Suppl. 76, 237 (1999).
[36] A. Gutiérrez-Rodríguez, M. A. Hernández-Ruíz, and L. N. Luis-Noriega, J. Phys. Conf. Ser. 37, 25 (2006).

[37] M. Passera, Nucl. Phys. B, Proc. Suppl. 169, 213 (2007).

[38] M. Passera, Phys. Rev. D 75, 013002 (2007).

[39] J. Bernabeu, G. A. González-Sprinberg, J. Papavassiliou, and J. Vidal, Nucl. Phys. B790, 160 (2008).

[40] A. Gutiérrez-Rodríguez, M. A. Hernández-Ruíz, C. P. Castañeda-Almanza, A. Espinoza-Garrido, and A. Chubykalo, Nucl. Phys. B, Proc. Suppl. 253-255, 202 (2014).

[41] W. Bernreuther, O. Nachtmann, and P. Overmann, Phys. Rev. D 48, 78 (1993).

[42] J. Abdallah et al. (DELPHI Collaboration), Eur. Phys. J. C 35, 159 (2004).

[43] M. Acciarri et al. (L3 Collaboration), Phys. Lett. B 434, 169 (1998).

[44] K. Ackerstaff et al. (OPAL Collaboration), Phys. Lett. B 431, 188 (1998).

[45] K. Inami et al. (BELLE Collaboration), Phys. Lett. B 551, 16 (2003).

[46] H. Albrecht et al. (ARGUS Collaboration), Phys. Lett. B 485, 37 (2000).

[47] J. A. Grifols and A. Méndez, Phys. Lett. B 255, 611 (1991); 259, 512(E) (1991).

[48] R. Escribano and E. Massó, Phys. Lett. B 395, 369 (1997).

[49] G. A. González-Sprinberg, A. Santamaria, and J. Vidal, Nucl. Phys. B582, 3 (2000).

[50] D. J. Silverman and G. L. Shaw, Phys. Rev. D 27, 1196 (1983).

[51] F. del Aguila and M. Sher, Phys. Lett. B 252, 116 (1990).

[52] L. Linssen et al., Report No. CERN-2012-003, 2012.

[53] E. Accomando et al. (CLIC Physics Working Group Collaboration), arXiv:hep-ph/0412251; Report No. CERN-2004-005.

[54] H. Abramowicz et al., arXiv:1307.5288.

[55] D. Dannheim et al., arXiv:1208.1402.

[56] A. Gutiérrez-Rodríguez, M. Koksal, and A. A. Billur, Phys. Rev. D 91, 093008 (2015).

[57] M. Köksal and S. C. Inan, Adv. High Energy Phys. 2014, 315826 (2014).

[58] M. Köksal, Mod. Phys. Lett. A 29, 1450184 (2014).

[59] C. Giunti and A. Studenkin, Rev. Mod. Phys. 87, 531 (2015).

[60] B. Grzadkowski, M. Iskrzynski, M. Misiak, and J. Rosiek, J. High Energy Phys. 10 (2010) 085.

[61] M. Fael, Ph.D. thesis, Universita Degli Studi Di Padova, 2014.

[62] S. Eidelman, D. Epifanov, M. Fael, L. Mercolli, and M. Passera, J. High Energy Phys. 03 (2016) 140.

[63] A. Belyaev, N. D. Christensen, and A. Pukhov, Comput. Phys. Commun. 184, 1729 (2013).

[64] I. F. Ginzburg et al., Nucl. Instrum. Methods Phys. Res. 219, 5 (1984).

[65] V. I. Telnov, Nucl. Instrum. Methods Phys. Res., Sect. A 294, 72 (1990).

[66] I. Sahin and M. Koksal, J. High Energy Phys. 03 (2011) 100.

[67] M. L. Perl et al., Phys. Rev. Lett. 35, 1489 (1975).

[68] M. L. Perl, Rep. Prog. Phys. 55, 653 (1992).

[69] H. Abramowicz et al., arXiv:1307.5288.

[70] A multi-TeV linear collider based on CLIC technology: CLIC conceptual design, edited by M. Aicheler et al., Report No. JAI-2012-001, KEK Report No. 2012-1, Report No. PSI-12-01, Report No. SLAC-R-985, https://edms.cern .ch/document/1234244/. 
[71] Physics and detectors at CLIC: CLIC conceptual design report, edited by L. Linssen et al., Report No. ANL-HEP-TR12-01, Report No. CERN-2012-003, DESY Report No. 12008, KEK Report No. 2011-7 (2012), https://arxiv.org/abs/ 1202.5940.

[72] S. Atag and A. A. Billur, J. High Energy Phys. 11 (2010) 060.

[73] A. Kalinowski, Nucl. Phys. B Proc. Suppl. 189, 305 (2009).
[74] S. Lai et al. (ATLAS Collaboration), Report No. ATLPHYS-PROC-2009-126.

[75] T. H. Tran, V. Balagura, V. Boudry, J. C. Brient, and H. Videau, Eur. Phys. J. C 76, 468 (2016).

[76] P. Achard et al. (L3 Collaboration), Phys. Lett. B 585, 53 (2004).

[77] S. Chatrchyan et al. (CMS Collaboration), J. High Energy Phys. 01 (2012) 052. 